\title{
Host Lymphotoxin- $\beta$ Receptor Signaling Is Crucial for Angiogenesis of Metanephric Tissue Transplanted into Lymphoid Sites
}

\author{
Maria Giovanna Francipane, ${ }^{* \dagger}$ Bing Han, ${ }^{*}$ and Eric Lagasse*
}

From the McGowan Institute for Regenerative Medicine and Department of Pathology, * University of Pittsburgh, Pittsburgh, Pennsylvania; and the Ri.MED Foundation, ${ }^{\dagger}$ Palermo, Italy

Accepted for publication

August 20, 2019.

Address correspondence to Maria Giovanna Francipane, Ph.D., 450 Technology Dr., Ste. 333, Pittsburgh, PA 15219; or Eric Lagasse, Pharm.D., Ph.D., 450 Technology Dr., Ste. 300, Pittsburgh, PA 15219. E-mail: francipa@pitt.edu or lagasse@ pitt.edu.

\begin{abstract}
The mouse lymph node (LN) can provide a niche to grow metanephric kidney to maturity. Here, we show that signaling through the lymphotoxin- $\beta$ receptor (LT $\beta R$ ) is critical for kidney organogenesis both in the $L N$ and the omentum. By transplanting kidney rudiments either in the LNs of mice undergoing LT $\beta R$ antagonist treatment or in the omenta of $L t b r$ knockout $\left(\mathrm{Ltbr}^{-/-}\right)$mice, the host LT $\beta \mathrm{R}$ signals were found to be crucial for obtaining a well-vascularized kidney graft. Indeed, defective LT $\beta R$ signaling correlated with decreased expression of endothelial and angiogenic markers in kidney grafts as well as structural alterations. Because the number of glomerular endothelial cells expressing the LT $\beta R$ target nuclear factor $\kappa B$-inducing kinase (NIK) decreased in the absence of a functional LT $\beta R$, it was speculated that an LT $\beta R /$ NIK axis mediated the angiogenetic signals required for successful ectopic kidney organogenesis, given the established role of NIK in neovascularization. However, the transplantation of kidney rudiments in omenta of $\mathrm{Nik}^{-/-}$mice revealed that NIK is dispensable for ectopic kidney vascular integration and maturation. Finally, defective LT $\beta R$ signaling impaired compensatory glomerular adaptation to renal mass reduction, indicating that kidney regeneration approaches, besides whole kidney reconstruction, might benefit from the presence of LT $\beta R$ signals. (Am J Pathol 2020, 190: 252-269; https://doi.org/10.1016/j.ajpath.2019.08.018)
\end{abstract}

Chronic and end-stage kidney disease impose a large burden on human health worldwide. Projected prevalence trend lines are not comforting, ${ }^{1}$ suggesting an urgent need to explore new approaches to overcome limitations associated with traditional interventions. Dialysis - the most common method used to treat advanced kidney failure-can only replace $10 \%$ to $20 \%$ of kidney function and is associated with increased left ventricular mass, intramyocardial cell fibrosis, and capillary loss., ${ }^{2,3}$ Orthotopic kidney transplantation offers better quality of life and longer survivals, ${ }^{4}$ yet it is limited by organ shortage and the risks associated with immunosuppression, including increased vulnerability to infection and cancer, as well as organ toxicity.

One novel solution to the shortage of human organs available for transplantation envisions growing new organs in situ via transplantation of developing anlagen from animal embryos. ${ }^{6}$ A few sites have been considered for renal anlage transplantation, and, paradoxically, the orthotopic environment seems not to be the most appropriate. Indeed, transplantation of metanephric tissue into the adult mouse kidney does not lead to glomerular or tubular differentiation. ${ }^{7}$ Other sites have also been considered, including the anterior eye chamber, ${ }^{8}$ the omentum, ${ }^{9}$ the paraaortic area, ${ }^{10}$ and, recently, the lymph node (LN) has been identified as an attractive site for the maturation of mouse metanephroi into functional glomeruli and tubules. ${ }^{11,12}$

Here, we show that host lymphotoxin- $\beta$ receptor (LT $\beta R$ ) pathway generates angiogenic cues to support kidney organogenesis in both LNs and omenta. Our hypothesis that

Supported by the Ri.MED Foundation (M.G.F.); NIH grant R01 DK085711 (E.L.); and The Pediatric Device Initiative-McGowan Institute for Regenerative Medicine, University of Pittsburgh - Commonwealth of Pennsylvania grant SAP4100073573 (E.L., M.G.F.).

Disclosures: E.L. is the scientific founder and the Chief Scientific Officer of LyGenesis, Inc. 
LT $\beta R$ could affect kidney organogenesis in these sites sprang from the central role of LT $\beta R$ in the development ${ }^{13,14}$ and homeostasis ${ }^{15}$ of lymphoid organs, as well as from our finding that glomeruli maturing in both LNs and omenta contained, during the early stages of engraftment, endothelial cells expressing the nuclear factor $\kappa \mathrm{B}$-inducing kinase (NIK), a downstream target, among others, of LT $\beta R$. $^{16}$ Given an established role for NIK in neovascularization, ${ }^{17}$ and the presence of host endothelial cells in ectopic kidney grafts, it was speculated that a host LT $\beta \mathrm{R} /$ NIK axis could allow successful kidney organogenesis. In accordance, defective LT $\beta$ R signaling was found to be associated with decreased numbers of glomerular NIK-expressing endothelial cells, decreased absolute vascularization/angiogenesis, and structural alterations of the kidney grafts. However, kidney organogenesis was not affected in the absence of NIK. This result might suggest either the existence of different targets downstream of LTRR, leading to angiogenesis, or the activation of compensatory angiogenic pathways in the absence of NIK.

Finally, the absence of LT $\beta$ R signals impaired compensatory glomerular adaptation to renal mass reduction, indicating that kidney regeneration approaches, besides whole kidney reconstruction, might benefit from the presence of LT $\beta R$ signals.

\section{Materials and Methods}

\section{Ethics Statement}

Mice were purchased from Charles River Laboratories (Wilmington, MA) or The Jackson Laboratory (Bar Harbor, $\mathrm{ME}$ ), and bred and housed in the Division of Laboratory Animal Resources facility at the University of Pittsburgh. Experimental protocols followed NIH's Guide for the Care and Use of Laboratory Animals, ${ }^{18}$ and they were approved by the University of Pittsburgh Institutional Animal Care and Use Committee. Mixed sexes were used.

\section{Tissues and Transplantations}

E14 kidneys were retrieved from timed-pregnant green fluorescent protein $(\mathrm{GFP})^{+}$or wild-type $\mathrm{C} 57 \mathrm{BL} / 6$ mice under a dissecting microscope. $\mathrm{GFP}^{+}$kidneys served for transplantation of wild-type C57BL/6 LNs to investigate host-derived vascularization (CD31 stain) and expression of LT $\beta R$, erasmus university rotterdam-thymic reticulum antibody 7 (ER-TR7), LT- $\beta$, and NIK ( $n=6)$ or to test the effect of LT $\beta \mathrm{R}-\mathrm{Fc}$ treatment $(n=6)$ (see below). Wild-type kidneys served for transplantation of wild-type C57BL/6 LNs $(n=3)$ to analyze glomerular NIK expression. LN transplantation was performed as previously described, ${ }^{11}$ and LN kidney grafts were collected 3 or 6 weeks after transplantation, depending on the experiment.

Alternatively, paired $\mathrm{GFP}^{+}$E14 kidneys were transplanted in the omenta of wild-type or $\mathrm{Ltbr}^{-/-} \mathrm{C} 57 \mathrm{BL} / 6$ mice $(n=10$ per group $)$ or in the omenta of wild-type or $\mathrm{Nik}^{-/-}$

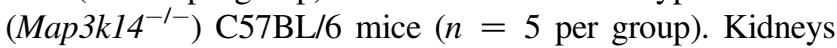
were split longitudinally in two pieces before transplantation. The kidney preparation was placed onto the exposed omentum, and the omentum was later folded onto itself. Growth Factor Reduced Matrigel (356231; Corning, Corning, NY) was used to glue the transplanted omenta. Intraomental kidney grafts from wild-type and $\mathrm{Ltbr}^{-/-}$mice were collected at different time points ( 2 weeks: $n=1 ; 3$ weeks: $n=4 ; 4$ weeks: $n=1 ; 5$ weeks: $n=1 ; 6$ weeks: $n=3$ ), whereas intraomental kidney grafts from wild-type and $\mathrm{Nik}^{-1-}$ mice were all collected 3 weeks after transplantation.

Wild-type or $\mathrm{Ltbr}^{-1-}$ C57BL/6 mice were also transplanted with $\mathrm{GFP}^{+}$newborn (postnatal day 2) kidneys in their omenta ( $n=3$ per group). Half of a kidney was transplanted in each mouse as described in the paragraph above. One mouse from each group was sacrificed every week, starting at 2 weeks after transplantation up to the fourth week.

Images of kidney grafts were obtained with both the Olympus IX71 inverted fluorescence microscope (Center Valley, PA) and the Leica EZ4D stereomicroscope (Buffalo Grove, IL).

\section{LT $\beta R$-Fc Treatments}

To assess LT $\beta$ R pathway contribution to kidney organogenesis inside the $\mathrm{LN}$, recipient mice were treated intraperitoneally with $100 \mu \mathrm{g}$ of mouse LT $\beta \mathrm{R}-\mathrm{Fc}$ fusion protein (71122; BPS Bioscience, San Diego, CA) or control immunoglobulin (PI31205; Pierce Biotechnology, Rockford, IL) 2 days before transplantation. Mice were further treated at day 8 and 15, and LNs were collected at day 22 . LT $\beta$ R-Fc concentration and exposure times were chosen based on previously published studies. ${ }^{19,20}$ LT $\beta$ R blockage was confirmed by decreased glomerular NIK expression.

\section{Stains and Quantifications}

Engrafted LNs and omenta and mouse kidneys were fixed in 4\% paraformaldehyde, embedded in optimal cutting temperature compound or paraffin, and cut into 4- to $7-\mu \mathrm{m}$-thick sections for further analysis. Hematoxylin and eosin (H\&E) staining was performed as described elsewhere. ${ }^{21}$ All other stains were performed as follows.

\section{Fluorescent Immunohistochemistry Staining of Frozen}

\section{Tissue Sections}

Frozen sections were equilibrated at room temperature for 15 minutes, then rehydrated in phosphate-buffered saline (PBS) for 15 minutes, and permeabilized with cold $0.1 \%$ Triton X-100 for 10 minutes. After three washes in PBS supplemented with $0.05 \%$ Tween 20 (PBS-T), a solution of $3 \%$ bovine serum albumin was applied to the sections for 30 minutes to prevent nonspecific binding. Sections were then incubated with specific primary 
antibodies at least 1 hour at room temperature or overnight at $4^{\circ} \mathrm{C}$. After three washes in PBS-T, sections were incubated with Alexa Fluor 488 or 594 secondary antibodies (Thermo Fisher, Pittsburgh, PA) for 20 minutes. Sections were washed three times, and the nuclei were counterstained with Hoechst 33342 (Life Technologies, Frederick, MD). A solution of 50\% glycerol in Hanks' balanced salt solution was used as mounting medium.

\section{Fluorescent Immunocytochemistry Staining}

CD45-depleted stromal cells from omenta were cytospun onto glass slides (see below for isolation), fixed with acetone for 8 minutes, and stained with specific antibodies according to the same protocol described for frozen sections.

\section{Chromogenic Immunohistochemistry Staining of Paraffin-} Embedded Tissue Sections

Paraffin sections were deparaffinized in xylene and rehydrated in graded alcohol. Endogenous peroxidases were blocked with the use of $3 \%$ hydrogen peroxide for $10 \mathrm{mi}-$ nutes. Antigen unmasking was performed with $10 \mathrm{mmol} / \mathrm{L}$ sodium citrate buffer $\mathrm{pH}$ 6.0. Nonspecific binding was prevented by incubating the slides in $3 \%$ bovine serum albumin in PBS-T for 30 minutes. The slides were exposed to primary antibodies overnight at $4^{\circ} \mathrm{C}$, incubated with biotinylated secondary antibody, and later with streptavidinhorseradish peroxidase (R.T.U. Vectastain Kit; Vector, Burlingame, CA). 3-Amino-9-ethylcarbazole (HK092-5KE; BioGenex, Fremont, CA) was used as a chromogen. Immunostained sections were counterstained with Mayer's hematoxylin (Dako, Carpinteria, CA) and mounted in permount mounting medium (SP15-500; Thermo Fisher).

A list of antibodies used is provided in Table 1. Isotypematched antibodies were used as negative controls. Images were obtained with the Olympus IX71 inverted fluorescence microscope.

\section{Fluorescent Quantifications}

Areas of LN and intraomental kidney grafts were quantified by measuring pixel counts of GFP signal with the use of Photoshop CS5 (Adobe, San Jose, CA). The mean fluorescence intensity (MFI) of podoplanin (PDPLN) and the fluorescence intensities of CD31, CD105, and Ly-76 were measured among regions of interest drawn around the Bowman capsule with the use of ImageJ version 1.52a (NIH, Bethesda, MD; https://imagej.nih.gov/ij), as previously described. ${ }^{22}$ Glomerular areas were quantified at the time of PDPLN MFI measurement. The corrected total glomerular fluorescence for CD31, CD105, and Ly-76 was later calculated with the following formula:

Integrated density - (area of selected glomerulus $\times$ mean fluorescence of background readings).

Length of lotus tetragonolobus lectin (LTL) ${ }^{+}$tubules was obtained with ImageJ version 1.52a by drawing a line over the $\mathrm{LTL}^{+}$stain (circular tubular structures were not
Table 1 List of Reagents Used in This Study

\begin{tabular}{lll}
\hline Antibody & $\begin{array}{l}\text { Catalog } \\
\text { number }\end{array}$ & Supplier \\
\hline CD105 & 550546 & BD Biosciences (San Jose, CA) \\
CD31 & ab28364 & Abcam (Cambridge, MA) \\
CD34 & MA1-82073 & ThermoFisher \\
CDH1 & sc-7870 & Santa Cruz Biotechnology (Dallas, TX) \\
CIV & $1340-01$ & SouthernBiotech (Birmingham, AL) \\
EFNB2 & ab131536 & Abcam \\
ER-TR7 & ab51824 & Abcam \\
Ki-67 & ab15580 & Abcam \\
LT- $\beta$ & ab64835 & Abcam \\
LT $\beta R ~$ & ab70063 & Abcam \\
LTL & B-1325 & Vector Laboratories (Burlingame, CA) \\
Ly-76 & ab91113 & Abcam \\
NIK & ab216409 & Abcam \\
PAI-1 & ab66705 & Abcam \\
PDPLN & NBP1-90211 & Novus Biologicals (Centennial, CO) \\
\hline
\end{tabular}

CDH1, cadherin 1; CIV, collagen IV; EFNB2, ephrin B2; ER-TR7, erasmus university rotterdam-thymic reticulum antibody $7 ;$ LT- $\beta$, lymphotoxin- $\beta$; $L T \beta R$, lymphotoxin- $\beta$ receptor; LTL, lotus tetragonolobus lectin; NIK, nuclear factor $\kappa \mathrm{B}$-inducing kinase; PAI-1, plasminogen activator inhibitor 1 ; PDPLN, podoplanin.

considered) and later converting the obtained numbers into microns. Glomeruli and tubules from at least five sections per sample were considered. ER-TR7 MFI was measured in 6 sections per sample with the use of ImageJ version 1.52a. The number of $\mathrm{NIK}^{+}$cells in $\mathrm{LN}$ and intraomental grafts was obtained by analyzing glomeruli from 7 to 13 microscopic fields depending on the experiment. The number of plasminogen activator inhibitor 1 (PAI-1) ${ }^{+}$tubules in LNs and intraomental grafts was obtained by analyzing 12 to 20 microscopic fields.

\section{Histologic Quantifications}

Quantitative measurement of glomerular diameters was performed by taking the maximum diameter from the hilum to the end of the Bowman capsule of $18 \mathrm{H} \& \mathrm{E}$-stained glomeruli derived from three sections per mouse, using the measurement tool in cellSens Standard 1.9 software (Olympus). Ki$67^{+}$nuclei were counted in at least five random sites within the cortex across two separate stained sections per mouse. The number of $\mathrm{Ki}-67^{+}$nuclei was finally expressed as a percentage of the total number of nuclei.

All sections were randomly selected and analyzed under blinded conditions (M.G.F., B.H.).

\section{Stromal Cell Isolation and Flow Cytometry}

Stromal cells were isolated from $\mathrm{GFP}^{+}$C57BL/6 mouse mesenteric LNs $(n=10)$ or omenta $(n=10)$ according to the method described by Turley laboratory. ${ }^{23}$ Briefly, tissues were collected and digested with an enzyme mix comprised of RPMI 1640 medium that contained $0.8 \mathrm{mg} / \mathrm{mL}$ dispase (17105-041; Gibco, Gaithersburg, MD), $0.2 \mathrm{mg} / \mathrm{mL}$ collagenase P (11213857001; Sigma-Aldrich, St. Louis, MO), 
and $1 \mathrm{mg} / \mathrm{mL}$ DNase I (89836; Thermo Fisher). After digestion, anti-CD45 immunomagnetic beads (130-052-301; Miltenyi Biotec, Auburn, CA) were used to deplete hematopoietic cells from the bulk population. CD45-depleted stromal cells were then stained with Sytox Blue (S11348; Molecular Probes, Eugene, OR), phycoerythrin-cyanine 7 CD45 (552848; BD Biosciences, Bedford, MA), phycoerythrin Podoplanin (127407; BioLegend, San Diego, CA), and peridinin chlorophyll protein/cyanine 5.5 CD31 (102419; BioLegend). Flow cytometric analysis was performed with a BD FACSAria cytometer and FlowJo software version 9.6 (TreeStar, Ashland, OR), according to standard procedures. Isotype antibodies were used as negative controls.

\section{Molecular Analysis}

RNA isolation was performed with the RNeasy Mini Kit (74106; Qiagen, Germantown, MD) according to the manufacturer's instructions (including the optional oncolumn DNase digestion). RNA purity and concentration were measured with a NanoDrop 2000/c spectrophotometer (Thermo Fisher). cDNAs were synthesized with the $\mathrm{RT}^{2}$ First Strand Kit (330401; Qiagen), and expression of 84 genes modulating the biological processes of angiogenesis were measured with the Mouse Angiogenesis $\mathrm{RT}^{2}$ Profiler PCR Array (PAMM-024Z; Qiagen) on a StepOnePlus Real-Time PCR System (Applied Biosystems, Foster City, CA), according to the manufacturer's

Table 2 List of Primer Sequences Used in This Study

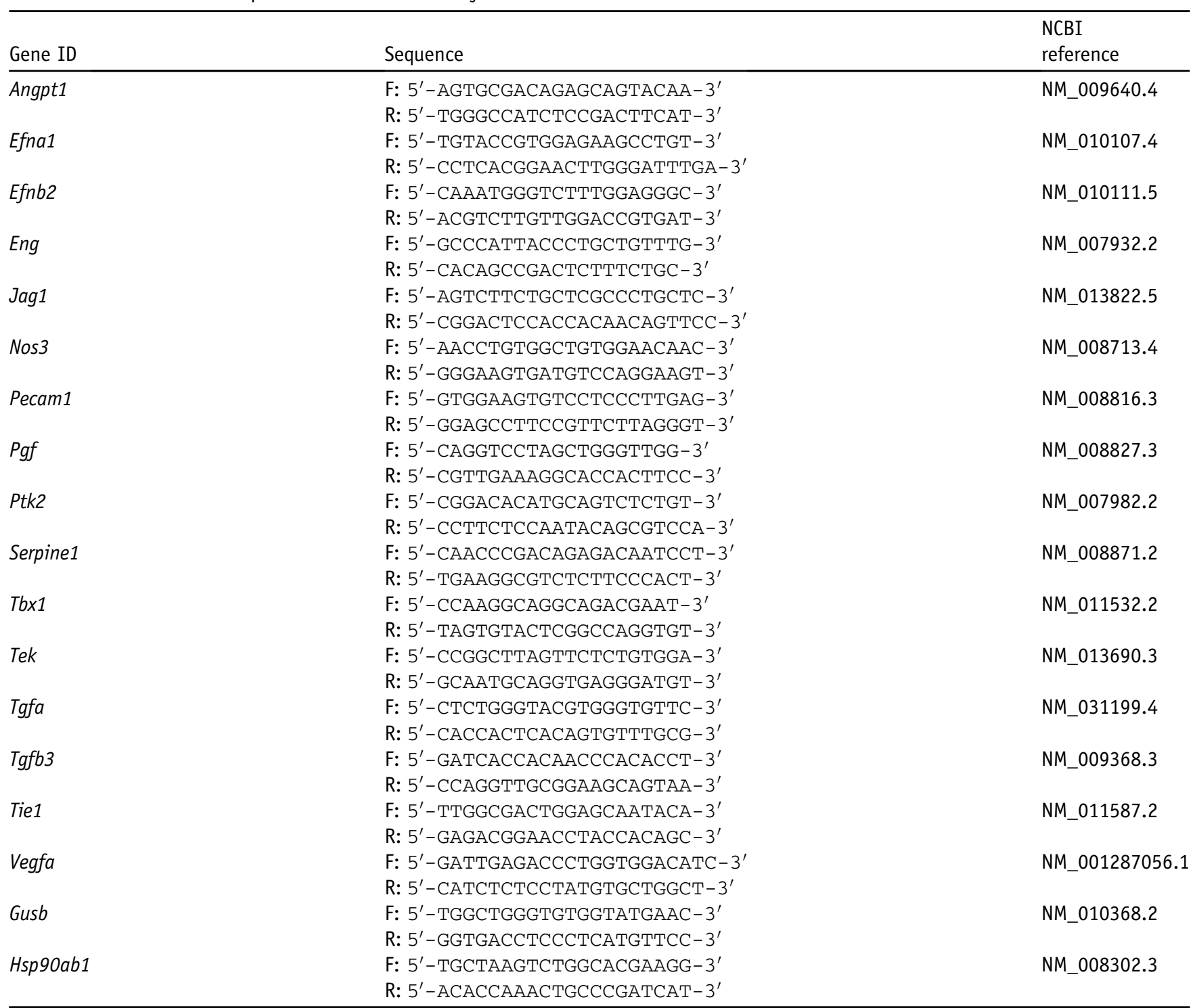

Primer sequences were designed with National Center for Biotechnology Information's (NCBI) Primer-BLAST (Basic Local Alignment Search Tool; https:// www.ncbi.nlm.nih.gov/tools/primer-blast, last accessed April 27, 2018). The NCBI Reference sequences that were entered in the Primer-BLAST submission form are indicated for each gene and were retrieved from NCBI's Nucleotide database (https://www.ncbi.nlm.nih.gov/refseq, last accessed April 27, 2018).

$F$, forward; $R$, reverse. 
instructions. The $\mathrm{C}_{\mathrm{T}}$ values from all plates and wells were then exported from the real-time PCR instrument and formatted into a new Excel spreadsheet (Microsoft, Redman, WA) (Supplemental Table S1). The data were uploaded in the $\mathrm{RT}^{2}$ Profiler PCR Array Data Analysis Webportal (https://dataanalysis.qiagen.com/pcr/ arrayanalysis.php, last accessed April 4, 2018) and analyzed. Gusb and Hsp90abl were automatically selected as optimal housekeeping genes. Genes with fold changes $>2$ and $P$ values $<0.05$ were later validated with real-time PCR. Primers were designed with NCBI's Primer-Blast (https://www.ncbi.nlm.nih.gov/ tools/primer-blast, last accessed April 27, 2018). Primers bridged an exon-exon junction or spanned introns. Free online Beacon Designer software version 7.9 (http://www.premierbiosoft.com/qOligo/Oligo.jsp?PID =1, last accessed April 27, 2018) was used to evaluate possible secondary structures such as dimers or hairpins.
To check the primer specificity, before performing real-time PCR, conventional PCR was performed, and amplicons were separated on agarose gels. A melting curve analysis was performed after each real-time PCR to further confirm that each assay produced a single, specific product. Primers sequences are given in Table 2.

Unilateral $\mathrm{Nx}$

Wild-type $(n=3)$ and $\mathrm{Ltbr}^{-1-}(n=4)$ C57BL/6 mice underwent unilateral nephrectomy $(\mathrm{Nx})$. After exposing the left kidney, the renal artery, vein, and ureter were tied off. The kidney was finally excised distal to the ligatures.

\section{Statistical Analysis}

Statistical analysis was performed with the unpaired $t$-test $\left(* P<0.05, * * P<0.01,{ }^{* * *} P<0.001\right.$,
A
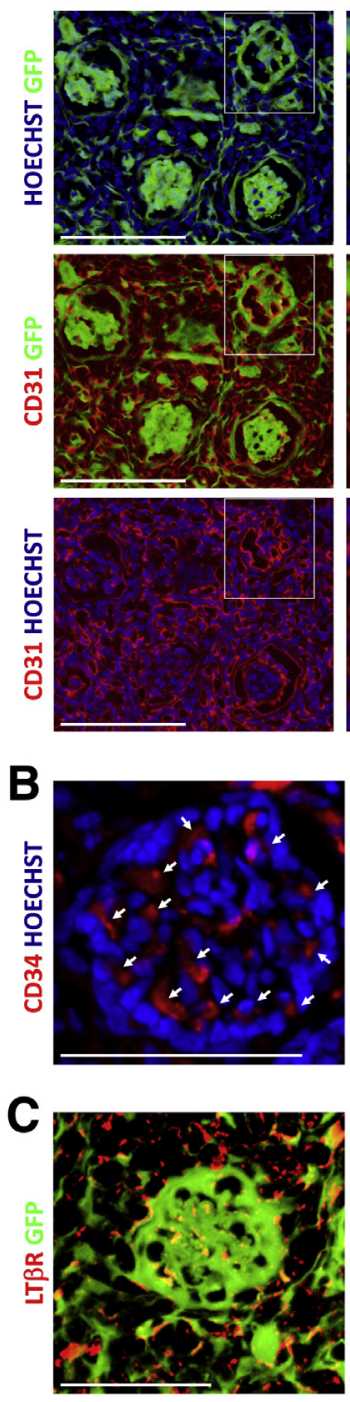
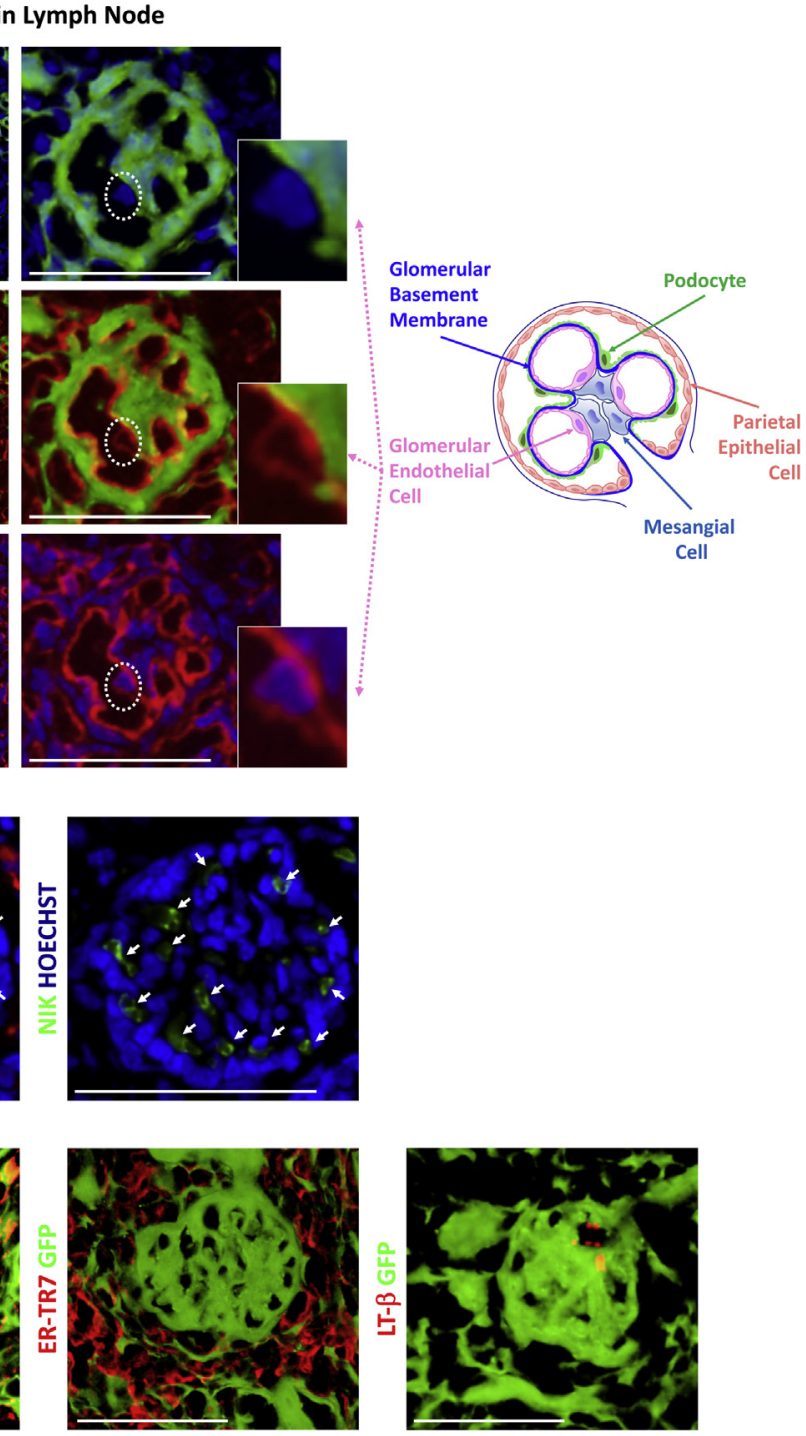

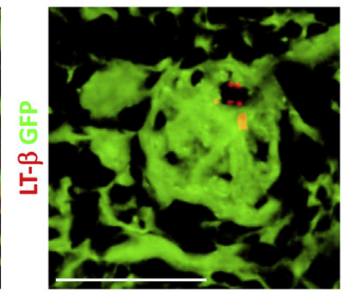

Figure 1 Vascularization of glomeruli engrafted in the mouse lymph node (LN) might be driven by host lymphotoxin- $\beta$ receptor $(\mathrm{LT} \beta \mathrm{R}) /$ nuclear factor $\kappa B$-inducing kinase (NIK) axis. A: Left column: Frozen sections of a representative green fluorescent protein (GFP) ${ }^{+}$metanephric (embryonic day 14) kidney graft 3 weeks after transplantation. Merged GFP signal with Hoechst stain (blue; top panel), merged GFP signal with CD31 (endothelial cell) stain (red; middle panel), and merged CD31 stain (red) with Hoechst stain (blue; bottom panel). Boxed and circled areas are shown at higher magnification to the right and demonstrate the presence of a host (GFP-) CD31 ${ }^{+}$ cell in an engrafted glomerulus. Right panel: Cartoon depicting the localization of glomerular endothelial cells (image adapted from http://whatwhen-how.com/acp-medicine/glomerular-diseasespart-1, last accessed June 1, 2019). B: Representative immunofluorescence stain for CD34 (endothelial cell marker, red) and NIK (green) in an engrafted wild-type (GFP') glomerulus 3 weeks after transplantation. Arrows point to double positive cells. C: Representative immunofluorescence stain for LT $\beta R$ (red), erasmus university rotterdam-thymic reticulum antibody 7 (ER-TR7; LN fibroblastic reticular cells, red), or lymphotoxin $\beta$ (LT- $\beta$, red) in engrafted $\mathrm{GFP}^{+}$ glomeruli 3 weeks after transplantation. Data presented in this figure are representative of one technical replicate of a minimum of three technical replicates per sample. $n=3$. Scale bars: $50 \mu \mathrm{m}$ (A, left column); $25 \mu \mathrm{m}$ (A, right column, $\mathbf{B}$, and $\mathbf{C}$ ). 
$* * * * P<0.0001)$. Statistical power analysis was also performed to assess error rates for tubule length measurements. For all studies, the power of a two-tailed $t$-test was $>0.9$, given an $\alpha$ level of $0.05(5 \%)$. A volcano plot of $\mathrm{RT}^{2}$ Profiler PCR Array data was used to identify genes differentially expressed in kidneys engrafted in $\mathrm{Ltbr}^{-/-}$versus wild-type omenta. Correlation between $\mathrm{RT}^{2}$ Profiler PCR Array and real-time PCR was calculated by linear regression and Pearson correlation. Both SABiosciences' data analysis (Frederick, MD) and GraphPad Prism 7 software (GraphPad Inc., San Diego, CA) were used.

\section{Results}

Vascularization of Glomeruli Engrafted in the Mouse LN Might Be Driven by Host LT $\beta R /$ NIK Axis

With the use of GFP-expressing mouse metanephric kidneys, successful nephron maturation inside a host mouse LN has been demonstrated. ${ }^{11,12}$ The LN-engrafted nephrons could rely on a remarkable vascular network originating from both host- and graft-derived vascular cells. ${ }^{11,12}$ Even more impressive were the data of human fetal kidney engraftment inside the LN, which clearly indicated that,

A

Days

\begin{tabular}{|c|c|c|c|}
\hline 13 & 8 & 15 & 22 \\
\hline Treatment & Treatment & Treatment & LNCollection \\
\hline
\end{tabular}

B

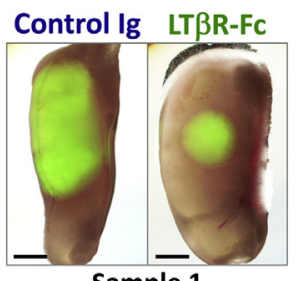

Sample 1
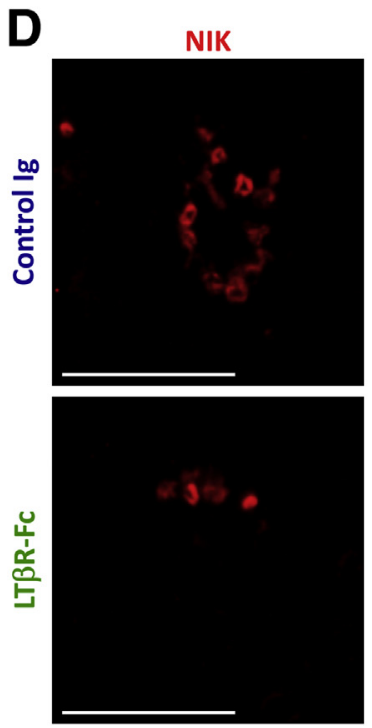

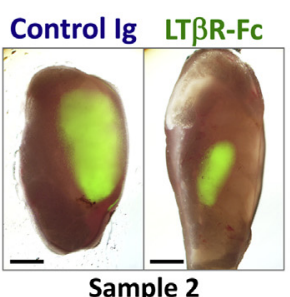

Sample 2
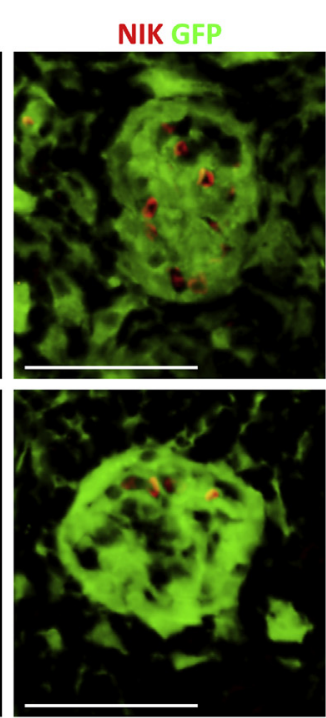

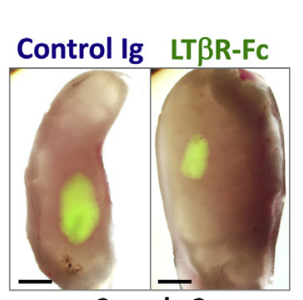

Sample 3

E
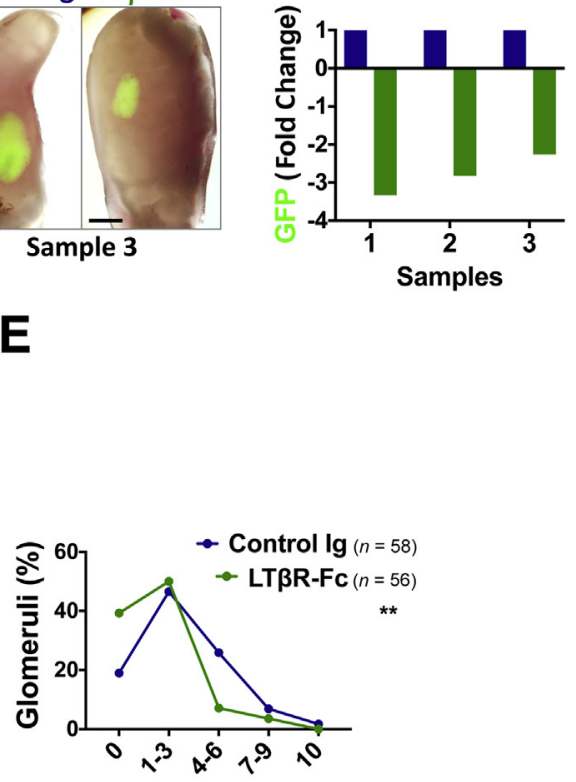

NIK Cells/Glom (Number)

Figure 2 Treatment with lymphotoxin- $\beta$ receptor antagonist (LT $\beta R-F c)$ impaired expansion of kidneys engrafted in lymph node (LN) and caused a decrease in nuclear factor $\kappa B$-inducing kinase (NIK)-expressing glomerular cells. A: Schematic representation of LN transplantation assay with the course of mouse treatments. Green K stands for green fluorescent protein (GFP) ${ }^{+}$metanephric (embryonic day 14) kidney. B: Merged fluorescence/bright-field images of whole mount kidney-bearing LNs isolated from either control Ig- or LT $\beta R$-Fc-treated mice (GFP ${ }^{+}$areas indicate kidney engraftment). C: Bar graph showing fold changes of GFP intensity (pixel counts obtained with Adobe Photoshop version CS5) in LNs as in B (blue bars: Control Ig; green bars: LT $\beta$ R-Fc). D: Immunofluorescence stain for NIK (red) in glomeruli of engrafted kidneys as in B. E: Line graph showing the percentage of NIK-expressing cells in engrafted glomeruli as in B. Glomeruli were divided into five groups based on the number of NIK-expressing cells $(0 ; 1$ to $3 ; 4$ to $6 ; 7$ to $9 ;$ or 10 positive cells/glomerulus). Data presented in $\mathbf{D}$ and $\mathbf{E}$ are representative of one technical replicate of a minimum of three technical replicates per sample. $n=3$ per group (D and $\mathbf{E})$. ${ }^{*} P<0.01 \mathrm{LT} \beta \mathrm{R}-\mathrm{Fc}$ versus control Ig (all glomeruli were considered regardless of the frequency of NIK-expressing cells). Scale bars: $500 \mu \mathrm{m}$ (B); $25 \mu \mathrm{m}$ (D). 
although the donor and host endothelial cells could coexist in the early stages of engraftment, over time the grafts were entirely re-vascularized by host cells. ${ }^{24}$

Figure 1A shows abundance of host $\left(\mathrm{GFP}^{-}\right)$endothelial cells $\left(\mathrm{CD} 31^{+}\right)$in the interstitium of a $\mathrm{GFP}^{+}$mouse metanephric kidney 3 weeks after transplantation in the LN, as well as the presence of a host endothelial cell in an engrafted glomerulus. Most glomerular endothelial cells $\left(\mathrm{CD} 34^{+}\right)$ expressed NIK (Figure 1B). Of interest, the number of $\mathrm{NIK}^{+}$ cells and/or NIK fluorescence intensity were significantly decreased in 6-week engrafted glomeruli (Supplemental Figure S1, A and B), suggesting that NIK expression could

A
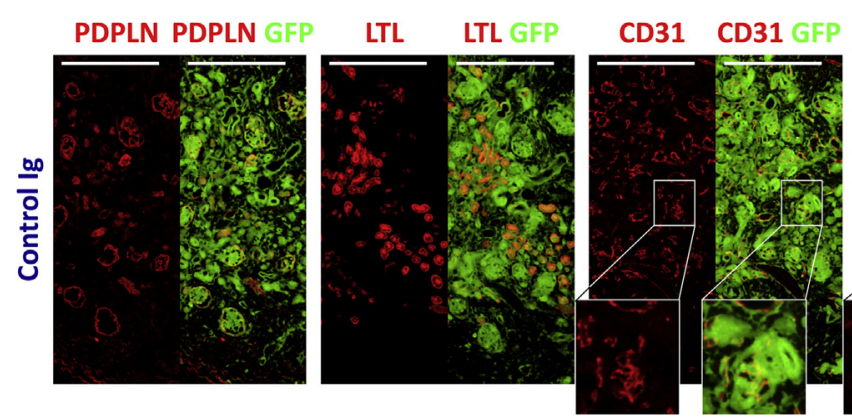

CD105 CD105 GFP
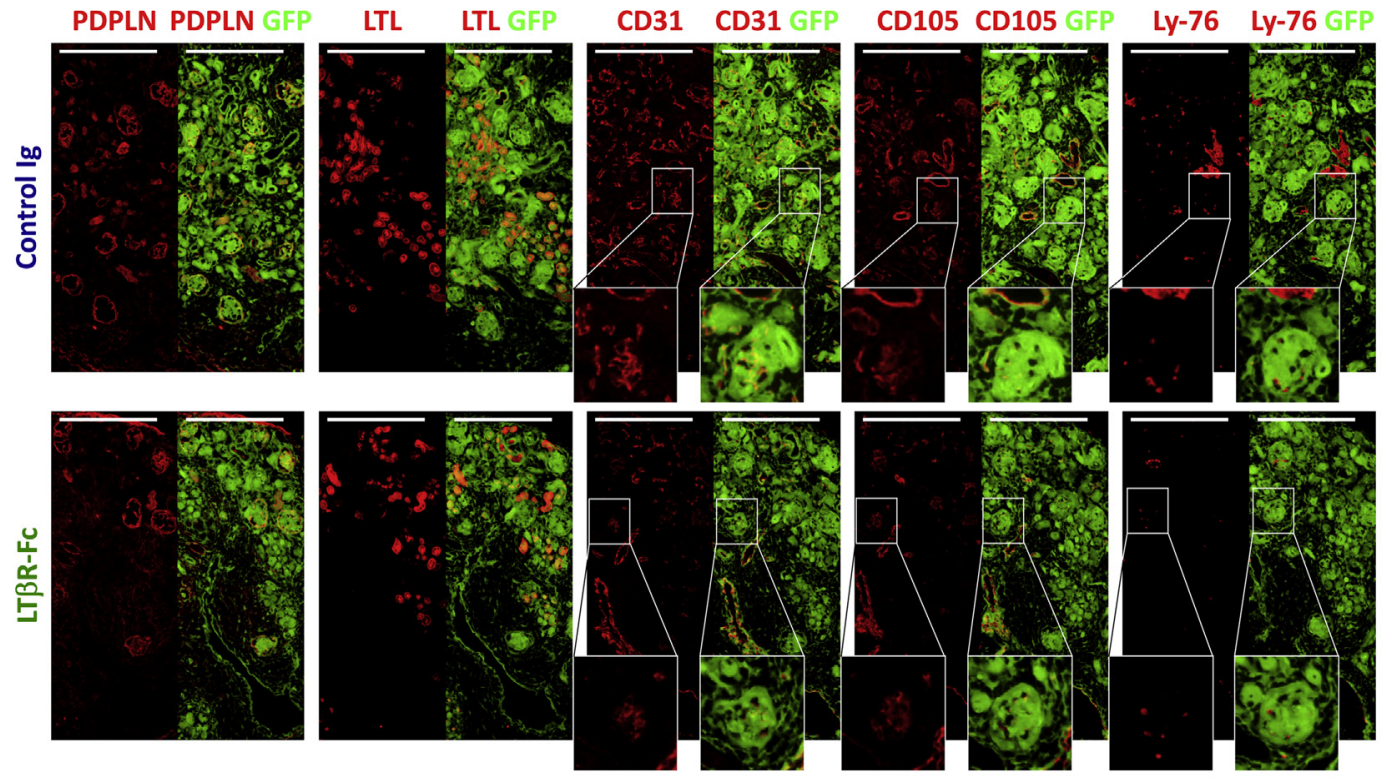

B
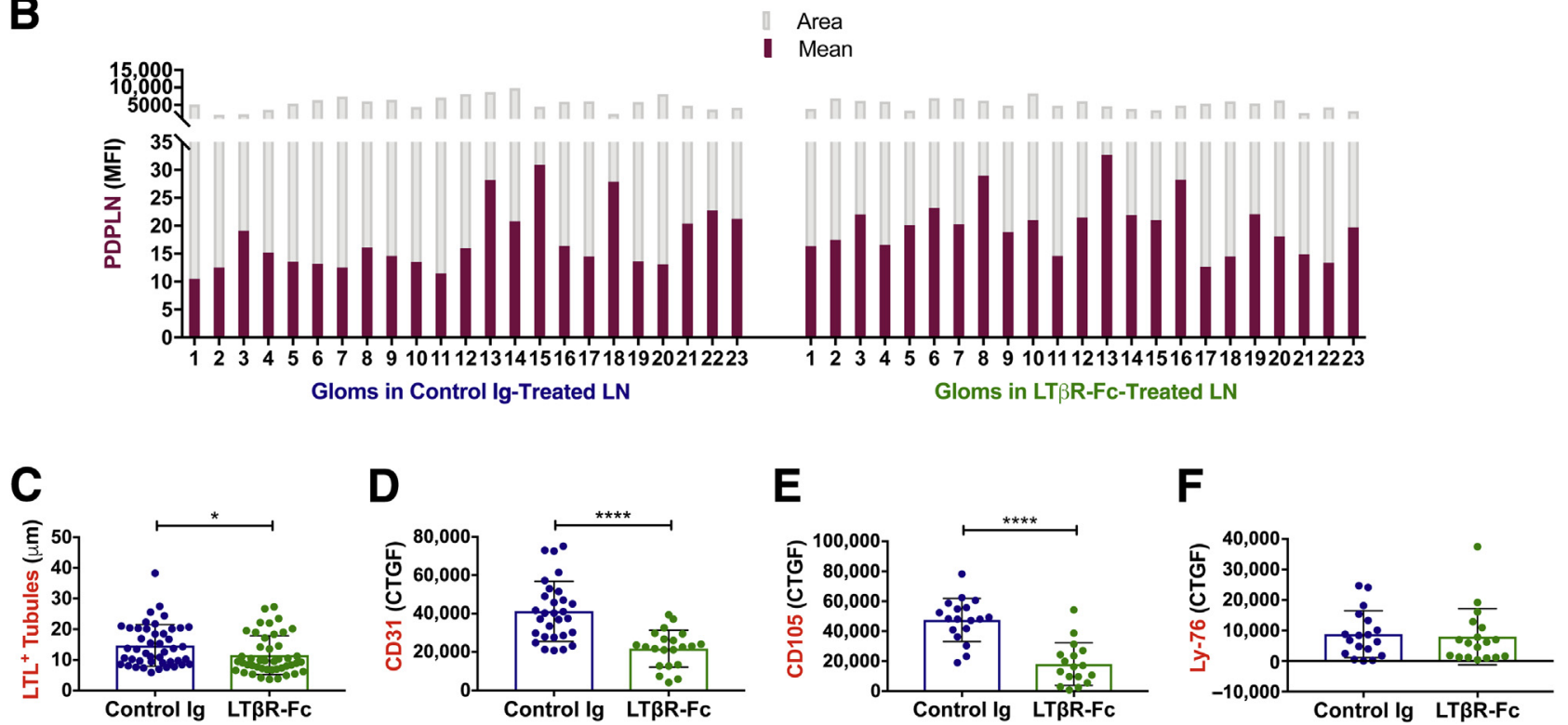

Figure 3 Treatment with lymphotoxin- $\beta$ receptor antagonist (LT $\beta R-F c)$ affected lymph node (LN) kidney graft architecture and vasculature. A: Representative immunofluorescence stains for podoplanin (PDPLN; podocytes and lymphatic endothelia, red), lotus tetragonolobus lectin (LTL; proximal tubules, red), CD31 (endothelial cells, red), CD105 (newly formed vessels, red), and Ly-76 (erythrocytes, red) on serial frozen sections of kidney grafts isolated from either Control Ig-or LTRR-Fc-treated mice. Insets show a representative glomerulus for each group. B: Bar graph showing PDPLN mean fluorescence intensity (MFI; plum bars) in glomeruli of kidney grafts as in $\mathbf{A}$, measured with ImageJ version 1.52a (each bar represents a glomerulus). Gray bars indicate the area of each glomerulus considered (ImageJ). C: Scatter dot plot with bar graphs \pm SD showing the length of LTL ${ }^{+}$tubules in kidney grafts as in $\mathbf{A}$, measured across sections, using ImageJ. Each dot represents a tubule. D-F: Scatter dot plot with bar graphs \pm SD presenting the corrected fluorescence intensities of glomerular (CTGF) CD31, CD105, and Ly-76 in kidney grafts as in A, measured with ImageJ. Each dot represents a glomerulus. Data presented in this figure are representative of one technical replicate of a minimum of three technical replicates per sample. $n=3$ per group. ${ }^{*} P<0.05,{ }^{* * * * P}<0.0001$. Power.t.test $=1$, sig.level $=0.05$ (C). Scale bars: $100 \mu \mathrm{m}(\mathbf{A})$. 
mark activated host endothelial cells during the early stages of kidney vascular integration in the host LN.

NIK is activated in response to stimulation of tumor necrosis factor receptor superfamily members such as LT $\beta \mathrm{R} .{ }^{25}$ LT $\beta R$ is widely expressed on the three major CD45 ${ }^{-} \mathrm{LN}$ stromal cell populations-fibroblastic reticular cells (FRCs), lymphatic endothelial cells, and blood endothelial cells - and is important for several adult LN functions. ${ }^{15}$ LT $\beta R$ signals on FRCs regulates LN vascular endothelial growth factor levels and, in turn, LN endothelial cell proliferation. ${ }^{26}$ Likewise, LT $\beta R$ triggering on LN endothelial cells affects vasculature development. ${ }^{27} \mathrm{We}$ therefore hypothesized that host LT $\beta$ R/NIK signaling facilitated glomerular vascularization inside the LN. In accordance with our hypothesis, engrafted glomeruli were found to be embedded in a host LT $\beta$ R reactive cellular scaffold, compatible with ER-TR7 ${ }^{+}$FRCs (Figure 1C). Intraglomerular LT $\beta$ R expression could also be observed, which, together with a rare intraglomerular expression of the membrane-anchored LT $\beta$ R ligand LT- $\beta$ (Figure 1C),
A

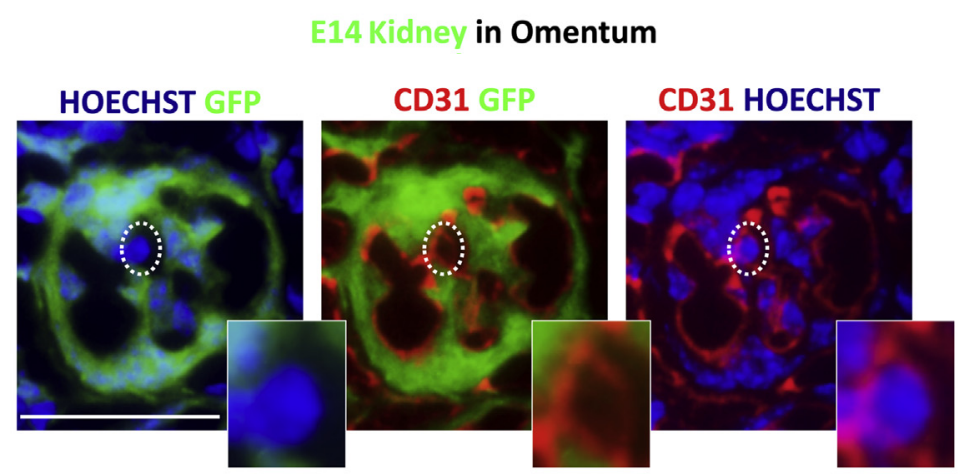

C

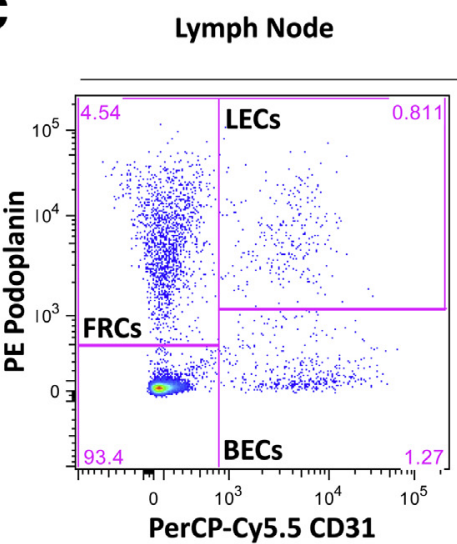

B
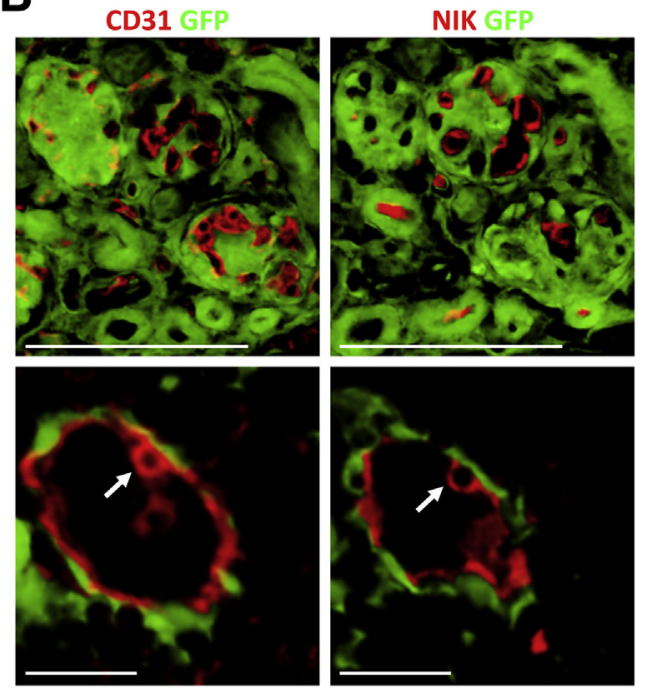

D

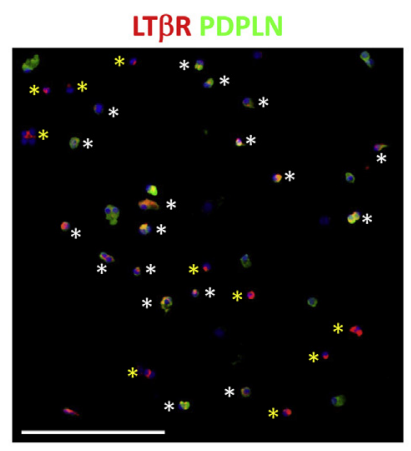

Figure 4 Metanephric kidney engraftment in omentum: similarity with the lymph node (LN) system. A: Frozen section of a representative green fluorescent protein (GFP) ${ }^{+}$glomerulus 3 weeks after transplantation in the omentum. Merged GFP signal with Hoechst stain (blue; left panel), merged GFP signal with CD31 (endothelial cell) stain (red; middle panel), and merged CD31 stain (red) with Hoechst stain (blue; right panel). Circled areas are shown at higher magnification below and demonstrate the presence of a host (GFP-) $\mathrm{CD}^{+} 1^{+}$cell in the engrafted glomerulus. B: Merged GFP signal with CD31 (red, left column) or nuclear factor $\mathrm{KB}$-inducing kinase (NIK; red, right column) stain in serial sections of $\mathrm{GFP}^{+}$kidney graft in the omentum ( 3 weeks after transplantation). Top row shows expressions of CD31 and NIK in glomeruli; bottom row, expressions of CD31 and NIK in a vessel. Arrows point to a host (GFP ${ }^{-}$) $\mathrm{CD}_{1} 1^{+} / \mathrm{NIK}^{+}$cell. C: Flow cytometric profiles of $\mathrm{CD} 45^{-}$cells isolated from LN (left panel) or omentum (right panel). From the podoplanin (PDPLN) and CD31 expression, distinct stromal cell populations can be identified: fibroblastic reticular cells (FRCs; PDPLN ${ }^{+} / \mathrm{CD}^{-} 1^{-}$), lymphatic endothelial cells (LECs; PDPLN ${ }^{+} /$ $\left(\mathrm{CD} 31^{+}\right)$, and blood endothelial cells (BECs; PDPLN $\left.{ }^{-} / \mathrm{CD} 31^{+}\right)$. D: Representative immunofluorescence stain for lymphotoxin- $\beta$ receptor (LT $\beta R ;$ red) and PDPLN (green) on $\mathrm{CD}_{4} 5^{-}$omental cells. White asterisks indicate $\mathrm{LT} \mathrm{R}^{+} / \mathrm{PDPLN}^{-}$cells and yellow asterisks indicate $\mathrm{LT} \beta \mathrm{R}^{+} / \mathrm{PDPLN}{ }^{+}$cells. Nuclei were counterstained with Hoechst (blue). Data presented in $\mathbf{A}$ and $\mathbf{B}$ are representative of one of three technical replicates per sample. $n=3$ (A and $\mathbf{B})$. Flow cytometric profiles and LT $\beta R / P D P L N$ stain were generated by pooling cells from 10 mice. Scale bars: $25 \mu \mathrm{m}$ (A); $50 \mu \mathrm{m}$ (B); $100 \mu \mathrm{m}$ (D). Cy, cyanine; PE, phycoerythrin; PerCP, peridinin chlorophyll protein. 
A

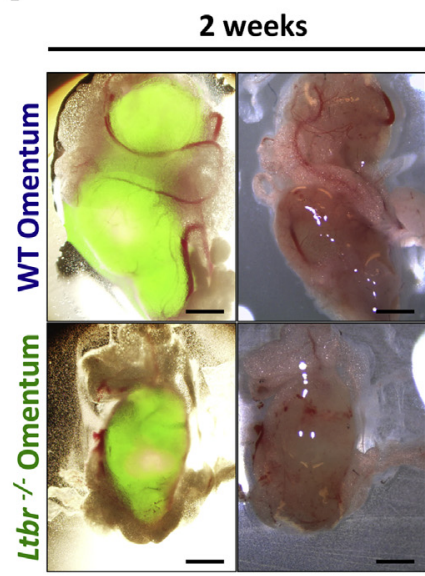

5 weeks

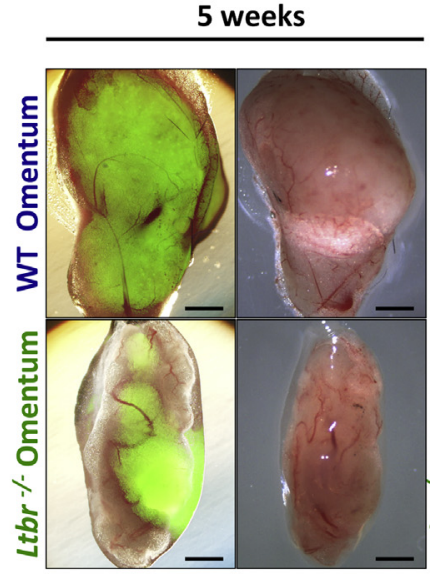

E14 Kidney in Omentum
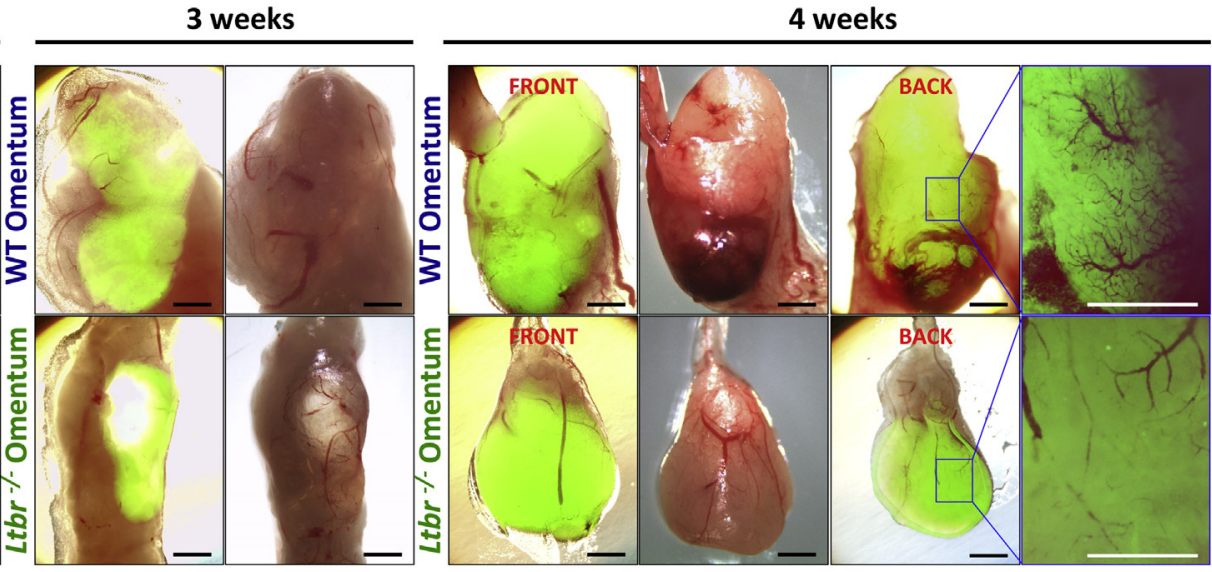

6 weeks
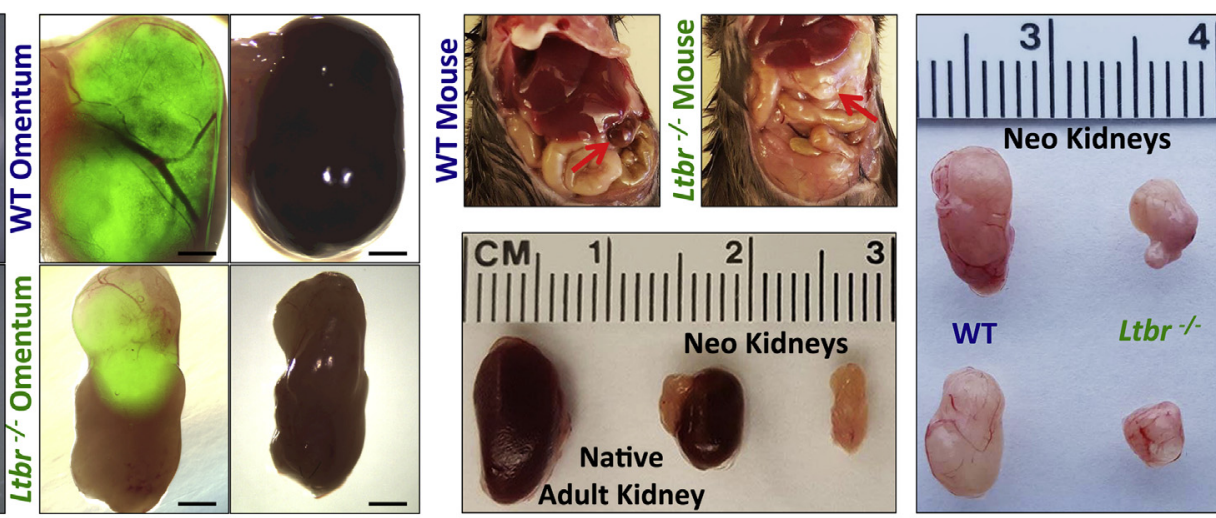

$\mathbf{B}$

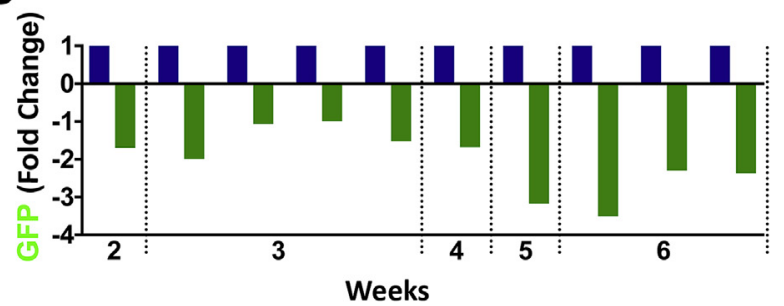

Figure 5 Defective lymphotoxin- $\beta$ receptor (LT $\beta R$ ) signaling in the omentum resulted in reduced kidney graft expansion. A: Merged fluorescence/brightfield (left panels) or dissecting scope (right panels) images of whole mount omenta from wild-type (WT) or Ltbr ${ }^{-/}$mice 2 to 6 weeks after transplantation of green fluorescent protein (GFP) ${ }^{+}$metanephric (embryonic day 14) kidneys. Grafts from 3 mice are shown at 6 weeks after transplantation in comparison with an adult mouse kidney. Red arrows point to the engrafted kidneys in WT or $\mathrm{Ltbr}^{-/-}$mice. B: Bar graph showing fold changes of GFP intensity (pixel counts obtained with Adobe Photoshop version CS5) in transplanted omenta as in A (blue bars: WT; green bars: Ltbr $^{-{ }^{-}}{ }^{-}$. Dotted lines separate the samples based on the time (weeks) elapsed from transplantation. $n=10$ per group; 2 weeks: $n=1$ per group; 3 weeks: $n=4$ per group; 4 weeks: $n=1$ per group; 5 weeks: $n=1$ per group; 6 weeks: $n=3$ per group. Scale bars: $500 \mu \mathrm{m}$ (main images); $250 \mu \mathrm{m}$ (enlargements).

strengthened the idea that the LT $\beta$ R/NIK axis could play a role in glomerular vascularization inside the LN.

\section{Inhibition of LT $\beta R$ Signaling Decreases Glomerular} Vascularization/Angiogenesis in the LN

To determine the role of LT $\beta$ R signaling on kidney engraftment in the LN, 2 days before metanephric kidney transplantation, recipient mice were treated with a recombinant LT $\beta \mathrm{R}-\mathrm{Fc}$ fusion protein-which antagonized LT $\beta$ R-mediated effects by engaging soluble and cell surface forms of LT $\beta R$ ligands - or control Ig (day 1). Mice were treated again at day 8 and 15, and LNs were collected at day 22 (Figure 2A). LT $\beta \mathrm{R}-\mathrm{Fc}$ treatment resulted in reduced expansion of transplanted $\mathrm{GFP}^{+}$metanephric kidneys (Figure 2, B and C) and decreased numbers of $\mathrm{NIK}^{+}$ glomerular cells (Figure 2D). Indeed, although glomeruli devoid of $\mathrm{NIK}^{+}$cells could be observed in both 
experimental groups, the number of such glomeruli was doubled in grafts from LT $\beta R-F c-$ treated mice (Figure 2E). Moreover, although both the groups had similar percentages of glomeruli that contained one to three $\mathrm{NIK}^{+}$cells, the number of glomeruli with four to six $\mathrm{NIK}^{+}$cells was drastically reduced after LT $\beta R-F c$ treatment (Figure 2E). Overall, glomeruli engrafted in LT $\beta \mathrm{R}-\mathrm{Fc}$-treated mice had a mean of $1.48 \pm 0.25 \mathrm{NIK}^{+}$cells, whereas glomeruli engrafted in control mice had a mean of $2.88 \pm 0.33 \mathrm{NIK}^{+}$ cells $(P=0.001)$.

When graft maturity was investigated through immunofluorescence staining for PDPLN to highlight the glomeruli, or LTL to visualize the proximal tubules, no obvious structural differences could be found between treated and control mice (Figure 3A). Further analysis confirmed that neither significant changes in total surface area of glomeruli nor significant changes in PDPLN expression had occurred (Figure 3B). Conversely, LT $\beta R-F c$ treatment resulted in decreased average $\mathrm{LTL}^{+}$tubule length, calculated in cross sections (Figure 3C). The extent of vascularization/angiogenesis was also evaluated by staining for CD31 and the neo-angiogenic marker CD105 (Figure 3A). The intraglomerular CD31 and CD105 fluorescence intensities were quantified, and a significantly weaker intensity was found for both markers in glomeruli of LT $\beta R-F c-$ treated mice (Figure 3, D and E). Nevertheless, no significant difference in glomerular blood perfusion was observed (Ly-76 stain), although fewer erythrocytes were located within blood vessels of treated grafts (Figure 3, A and F). Thus, LT $\beta$ R signaling might mediate vascularization/angiogenesis of the kidneys transplanted in the LN.

\section{Glomerular Vascularization/Angiogenesis Is Decreased in Kidneys Engrafted in the $\mathrm{Ltbr}^{-/}$Omentum}

The LT $\beta$ R ligands include the heterotrimeric LT $\alpha 1 \beta 2$ $(\mathrm{LT} \alpha 1 \beta 2)^{28}$ and LT-related inducible ligand. ${ }^{29}$ Although LT $\alpha 1 \beta 2$ binds uniquely to LT $\beta$ R, LT-related inducible ligand may also interact with herpesvirus entry mediator. ${ }^{30}$ To rule out the possibility that impaired graft angiogenesis resulted from LT $\beta R-F c$ interference with signaling pathways other than those mediated by LT $\beta R$, the outcome of kidney transplantation in an LT $\beta R$ defective environment, such as that offered by the $L t b r^{-/-}$mouse, was investigated. In addition, this approach allowed excluding the possibility that the observed effects could be due to LT $\beta R /$ herpesvirus entry mediator inhibition on donor kidney cells or other cell types present in the donor tissue at the time of transplantation, including stromal and hematopoietic cells. Because $L t b r^{-l-}$ mice do not have LNs ${ }^{13}$ the omentum was used as an alternative site for transplantation. Similarly to the LN, the omentum supported the maturation of GFPexpressing metanephric kidneys. Intraomental kidney grafts contained host-derived $\left(\mathrm{GFP}^{-}\right)$glomerular endothelial cells $\left(\mathrm{CD} 31^{+}\right)$(Figure 4A) as well as NIK-expressing endothelial cells $\left(\mathrm{CD} 31^{+}\right)$in renal glomeruli and blood vessels (Figure 4B). We therefore hypothesized that LN and omentum supported kidney organogenesis through a similar cellular/molecular mechanism. Our hypothesis was championed by the finding that both sites shared similar FRC/ lymphatic endothelial cell/blood endothelial cell flow cytometric profiles (Figure $4 \mathrm{C}$ ). Moreover, $\mathrm{LT} \beta \mathrm{R}^{+} / \mathrm{PDPLN}^{+}$ and $\mathrm{LT} \mathrm{R}^{+} / \mathrm{PDPLN}^{-}$cell subsets could be identified in omental cell suspensions, indicating the existence of FRCs and blood endothelial cells that mediated LT signals in this site as well (Figure 4D).

Consistent with our previous findings (Figure 2, B and C), expansion of $\mathrm{GFP}^{+}$metanephric kidneys was affected in the $\mathrm{Ltbr}^{-1-}$ omentum. Not only did the grafts expand less over a 6-week period (Figure 5), but they also were significantly less vascularized (Figure 6A). The glomerular CD31, CD105, and Ly-76 fluorescence intensities were quantified; significant changes could be observed at every time point analyzed (Figure 6B). Of note, no significant changes in Ly-76 expression had been observed in LN kidney grafts isolated from mice undergoing LT $\beta R-F c$ treatment with respect to their control counterparts (Figure 3F). The cause for this different outcome remains unknown.

CD31 and CD105 fluorescence intensities were also compared over time (Figure 6, C and D). CD31 expression was stable up to 4 weeks in glomeruli engrafted in the wildtype omentum and decreased afterward (Figure 6C). Conversely, CD31 expression began falling soon after the second week of transplantation in glomeruli engrafted in the $\mathrm{Ltbr}^{-/-}$omentum (Figure 6C). CD105 expression had a similar trend in both experimental groups, with the highest expression registered at 2 weeks after transplantation (Figure 6D). At this stage, significant differences in $\mathrm{NIK}^{+}$ glomerular cell numbers could also be observed between the two kidney groups (Supplemental Figure S1C and Figure 6E). Indeed, the number of glomeruli devoid of $\mathrm{NIK}^{+}$cells increased in the $\mathrm{Ltbr}^{-/-}$omentum (Figure 6E). Moreover, although almost an equal number of glomeruli had either one to three or four to six $\mathrm{NIK}^{+}$cells in the wildtype omentum, the number of glomeruli with one to three $\mathrm{NIK}^{+}$cells increased in the $\mathrm{Ltbr}^{-/}$omentum, to the detriment of glomeruli with four to six $\mathrm{NIK}^{+}$cells (Figure 6E). Finally, glomeruli with seven to nine $\mathrm{NIK}^{+}$ cells were more abundant in the wild-type omentum than in the $\mathrm{Ltbr}^{-/-}$omentum, and glomeruli with up to ten $\mathrm{NIK}^{+}$ cells could only be observed in the wild-type omentum (Figure 6E). Overall, glomeruli engrafted in the $\mathrm{Ltbr}^{-1-}$ omentum had a mean of $2.04 \pm 0.23 \mathrm{NIK}^{+}$cells, whereas glomeruli engrafted in the wild-type omentum had a mean of $4.04 \pm 0.30 \mathrm{NIK}^{+}$cells $(P<0.0001)$. Over the time course, the number of glomeruli devoid of $\mathrm{NIK}^{+}$cells progressively increased in both groups, and the number of $\mathrm{NIK}^{+}$cells per positive glomerulus also decreased (Supplemental Figure S1, D and E). Four weeks after transplantation, no more than three $\mathrm{NIK}^{+}$cells could be observed in glomeruli engrafted in the $L t b r^{-1-}$ omentum, 
A
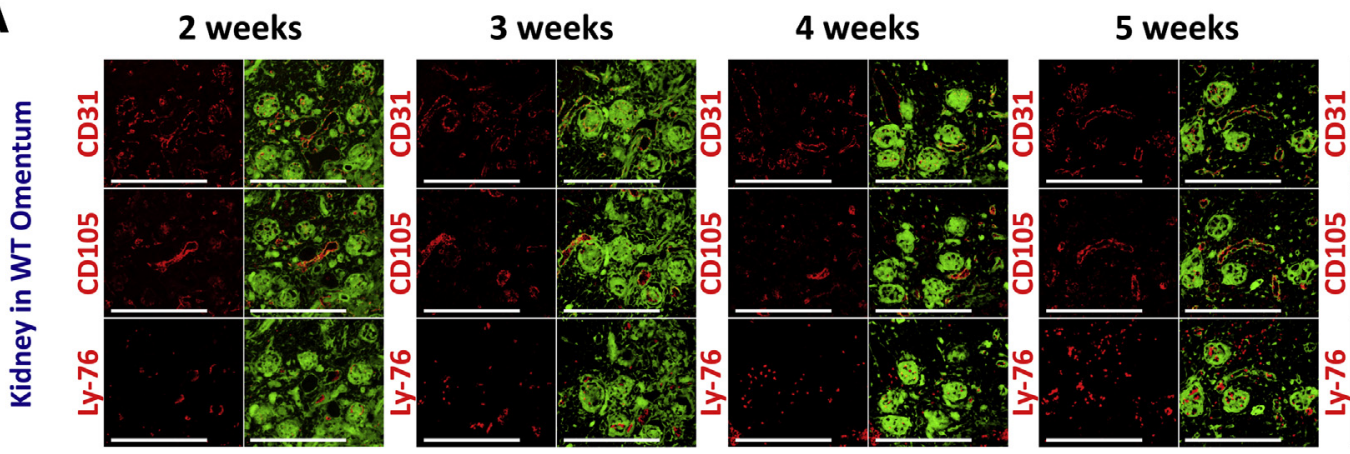

6 weeks
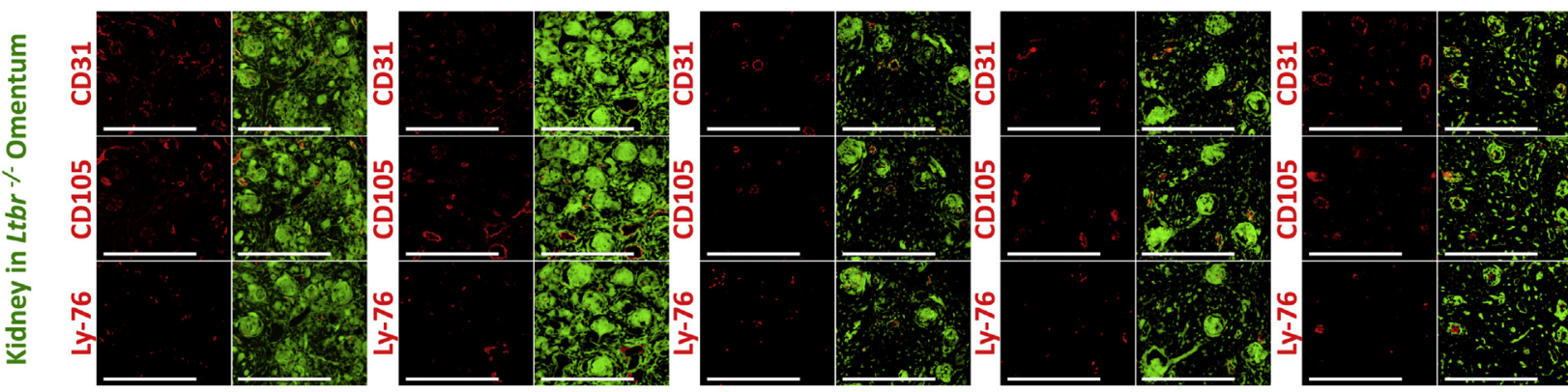

B

2 weeks

3 weeks

4 weeks

5 weeks

6 weeks
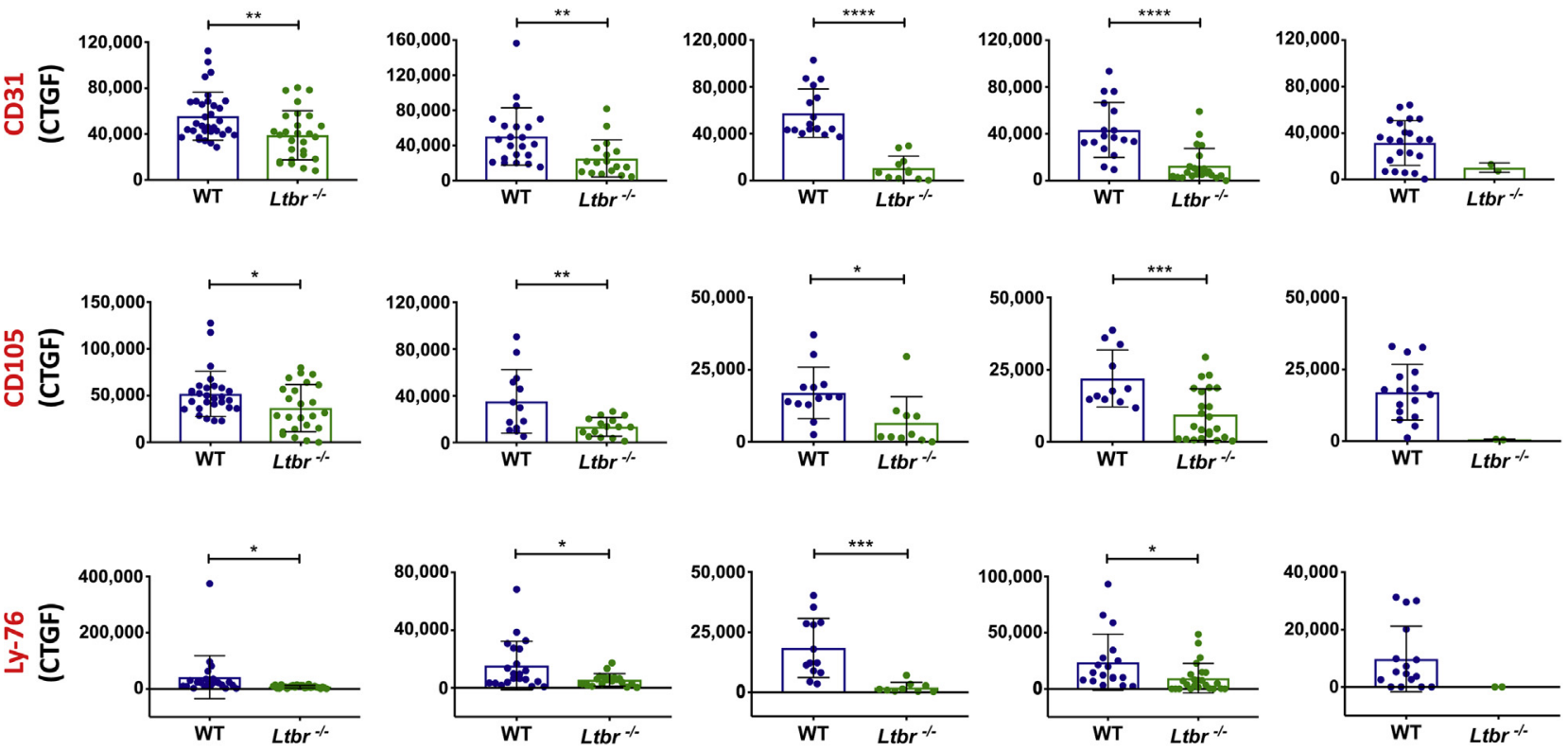

C

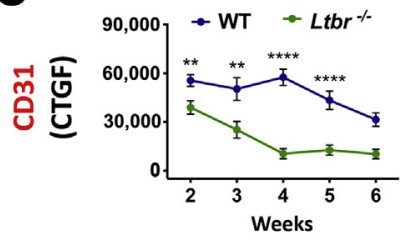

D

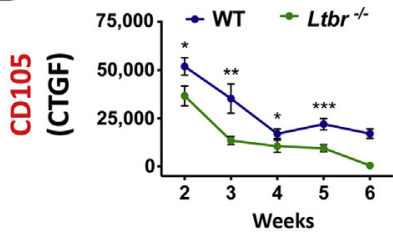

E

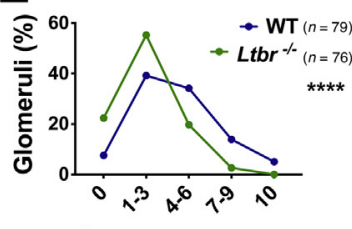

NIK ${ }^{+}$Cells/Glom (Number) 
whereas a small percentage of glomeruli engrafted in the wild-type omentum had four to six $\mathrm{NIK}^{+}$cells (Supplemental Figure S1, D and E). These results suggested a critical time window between 2 and 3 weeks after transplantation for LT $\beta$ R/NIK actions on the vascular remodeling of the transplanted glomeruli.

\section{Structural Changes in Kidneys Engrafted in the $\mathrm{Ltbr}^{-/-}$ Omentum}

With respect to their control counterparts, the kidneys engrafted in the $\mathrm{Ltbr}^{-/-}$omentum also showed structural changes overtime, with complete loss of glomeruli and tubules by 6 weeks (Figure 7A).

The time point of 2 weeks was specifically studied. Although no significant changes in total surface area of glomeruli were observed, highly significant changes affected PDPLN expression (Figure $7 \mathrm{~B}$ ) and $\mathrm{LTL}^{+}$tubule length (Figure 7C).

Tubulointerstitial injury has been described as a mediator of progression of kidney disease. ${ }^{31}$ It was therefore studied whether the observed glomerular and tubular loss in later stages of engraftment could be due to the expansion of renal interstitial fibroblasts. To test this hypothesis, staining was performed for ER-TR7, which besides outlining the various compartments of peripheral lymphoid organs by detecting the reticular fibroblasts, also identifies interstitial fibroblasts in the kidney (Supplemental Figure S2A). ${ }^{32}$ Significant changes were not observed between the kidneys engrafted in the wildtype omentum versus those engrafted in the $L t b r^{-1-}$ omentum over the 6-week period (Supplemental Figure S2B). Moreover, in each group, ER-TR7 levels were quite stable until the fifth week, after which time they decreased (Supplemental Figure S2C). Thus, the precise mechanism of glomerular and tubular loss in late-stage grafts remains unknown.

Similar results were obtained when using newborn kidneys, instead of metanephric kidneys (Supplemental Figure S3), indicating that host-driven angiogenesis was uncoupled from the maturation stage of the transplanted tissue (ie, ongoing versus completed angiogenesis). Of interest, when using newborn kidneys, more pronounced structural changes were detected between the two mouse groups, starting as early as the second week after transplantation (Supplemental Figure S3).
Thus, these results pinpointed the importance of host LT $\beta R$ signaling for successful vascularization/ angiogenesis and maturation of the kidney grafts in lymphoid sites.

\section{Reduced Expression of Angiogenesis-Related Genes in Kidneys Engrafted in the $\mathrm{Ltbr}^{-/-}$Omentum}

PCR array profiles of angiogenesis-related genes confirmed that host LT $\beta$ R signaling sustains ectopic kidney angiogenesis. Indeed, when six distinct transplants engrafted either in the $\mathrm{Ltbr}^{-/-}$omentum or in the wildtype omentum were compared (3 weeks after transplantation), several proangiogenic factors were found to be down-regulated in the absence of functional LT $\beta R$ signaling (Figure 8 and Supplemental Figure S4). These factors included components of the angiopoietin-Tie signaling system (Angpt1, Tek, and Tie1); the epidermal growth factor signaling system (Tgfa); the Eph-ephrin signaling system (Efnal and Efnb2); the transforming growth factor $\beta$ signaling system (Eng and Tgfb3); the vascular endothelial growth factor signaling system $(P g f)$; and targets shared among these systems (Jag1, Nos3, Ptk2, Serpine1, Tbxl).

Expression of Efnb2 and Serpinel products was later analyzed in sections of 3-week intraomental kidney grafts. EFNB2 was found to be expressed in glomerular cells (presumably podocytes and parietal epithelial cells), and PAI-1, the product of Serpinel, was expressed in renal tubules (presumably proximal tubules) (Figure 9A). Although EFNB2 fluorescence intensity was similar in both groups, a significant lower number of tubules were positive for PAI-1 in the kidneys engrafted in the $L t b r^{-1-}$ omentum (Figure 9B). A similar trend could be observed in kidneys engrafted in LNs under LT $\beta R-F c$ treatment (Figure 9, C and D), confirming that a similar cellular/ molecular mechanism drove kidney organogenesis in LNs and omenta. These results also suggested that, although the lymphoid microenvironment provided signaling cues that affected the vasculature of the maturing kidney, the kidney graft self-sustained by producing angiogenic molecules. Production of these molecules appeared to be facilitated by an environment that mediated LT signals.

\footnotetext{
Figure 6 Defective lymphotoxin- $\beta$ receptor (LT $\beta R$ ) signaling in the omentum resulted in reduced kidney graft vascularization/angiogenesis and decreased numbers of nuclear factor $\mathrm{\kappa B}$-inducing kinase (NIK)-expressing glomerular cells. A: Representative immunofluorescence stains for CD31 (endothelial cells, red), CD105 (newly formed vessels, red), and Ly-76 (erythrocytes, red) on serial frozen sections of intraomental kidney grafts from wild-type (WT) or Ltbr ${ }^{-/}$ mice over the course of 6 weeks. B: Scatter dot plot with bar graphs \pm SD presenting the corrected fluorescence intensities of intraglomerular (CTGF) CD31, CD105, and Ly-76 in kidney grafts as in A, measured with ImageJ version 1.52a. Each dot represents a glomerulus. C and D: Line graphs \pm SEM presenting CD31 and CD105 CTGFs over the time course (blue line: WT; green line: $\mathrm{Ltbr}^{-{ }^{-}}$). E: Line graph showing the percentage of NIK-expressing cells in glomeruli 2 weeks after transplantation in WT (blue line) or $\mathrm{Ltbr}^{-/-}$(green line) omentum. Glomeruli were divided into five groups based on the number of NIK-expressing cells $(0 ; 1$ to $3 ; 4$ to $6 ; 7$ to 9 ; or 10 positive cells/glomerulus). Data presented in this figure are representative of one of three technical replicates per sample. $n=10$ per group; 2 weeks: $n=1$ per group; 3 weeks: $n=4$ per group; 4 weeks: $n=1$ per group; 5 weeks: $n=1$ per group; 6 weeks: $n=3$ per group. ${ }^{*} P<0.05,{ }^{* *} P<0.01,{ }^{* * *} P<0.001,{ }^{* * *} P<0.0001 \mathrm{Ltbr}^{-/-}$versus WT (in $\mathbf{E}$, all glomeruli were considered regardless of the frequency of NIK-expressing cells). Scale bars $=100 \mu \mathrm{m}(\mathbf{A})$.
} 
Host NIK Is Not Required for Ectopic Kidney Organogenesis

A significant reduction in the number of NIK-expressing glomerular cells had been observed in kidneys engrafted in both LNs of mice undergoing LT $\beta$ R antagonist treatment (Figure 2, D and E) and omenta of $L t b r^{-/-}$mice (Figure 6E and Supplemental Figure S1, C-E). To elucidate whether a functional NIK was required for successful ectopic kidney organogenesis, $\mathrm{GFP}^{+}$metanephric kidneys were transplanted in wild-type or $\mathrm{Nik}^{-/-}$omenta. Significant differences in graft size, structure, vascularization, or angiogenesis were not observed at 3 weeks after transplantation (Supplemental Figure S5, A-K), indicating that, although NIK could be activated by LT $\beta$ R signaling in this system, it was dispensable for ectopic kidney organogenesis. Moreover, NIK-expressing cells could still be detected in kidneys engrafted in the $\mathrm{Nik}^{-/}$omentum 3 weeks after transplantation (Supplemental Figure S6), although NIK fluorescence intensity was much lower than that observed in
A
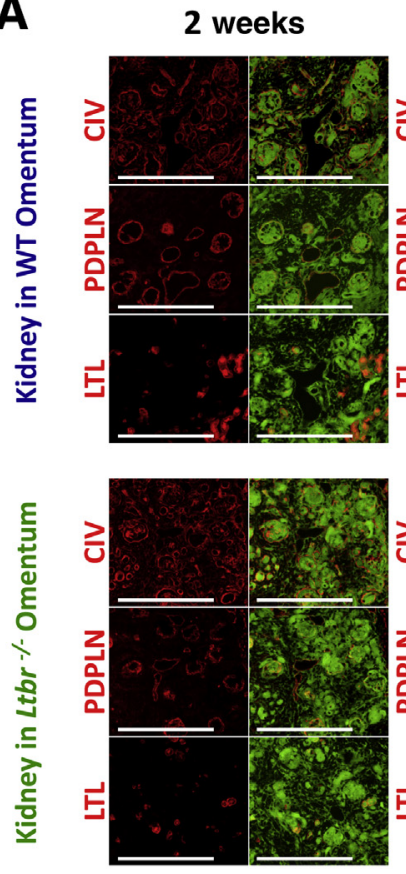

B

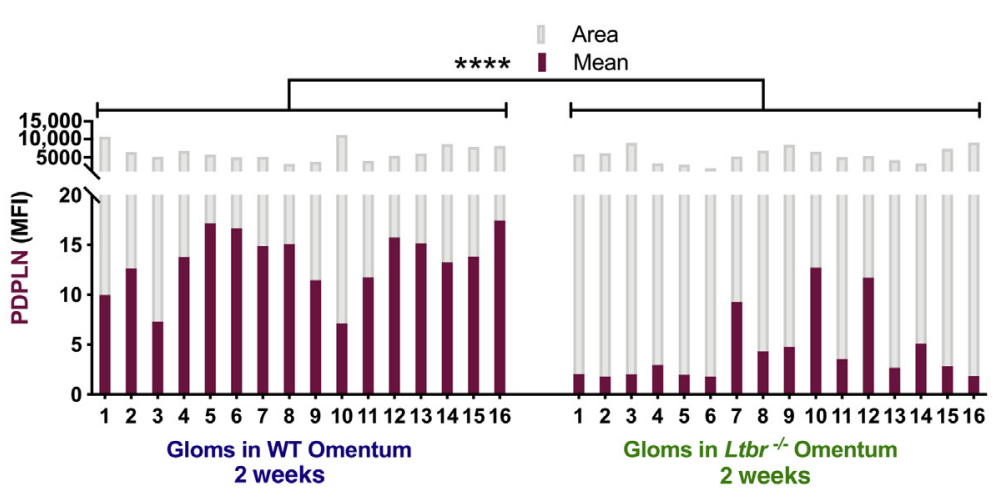

5 weeks
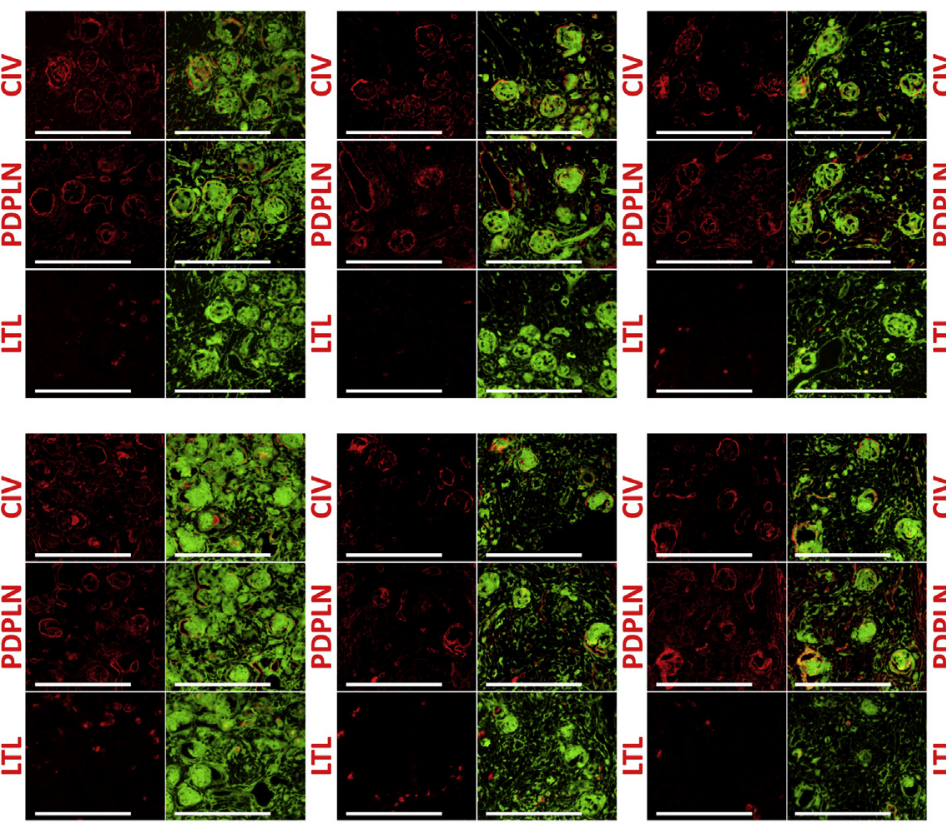

C
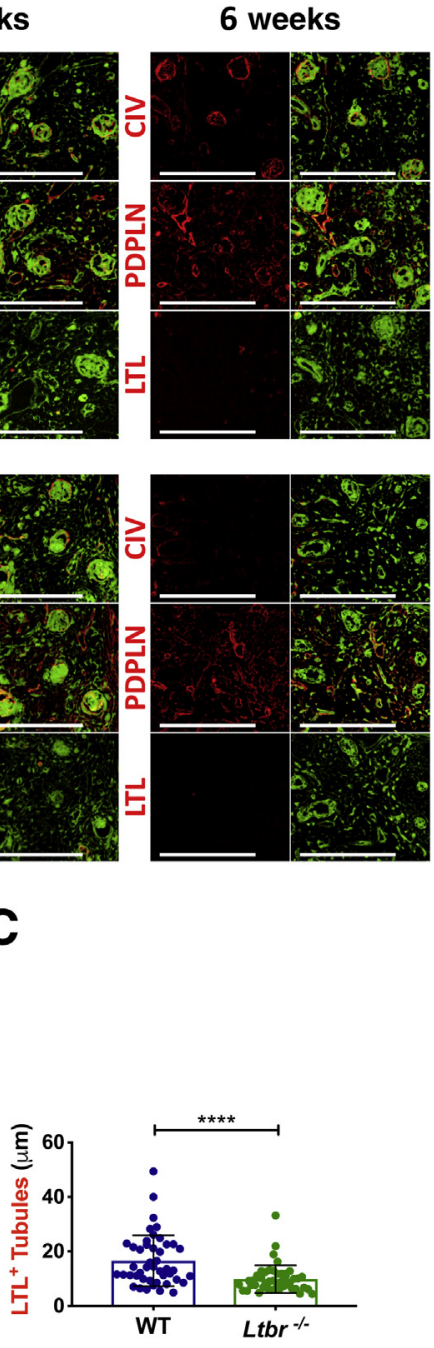

Figure 7 Defective lymphotoxin- $\beta$ receptor (LT $\beta R$ ) signaling in the omentum affected kidney graft architecture. A: Representative immunofluorescence stains for collagen IV (CIV; glomerular basement membranes and mesangial cells, red), podoplanin (PDPLN; podocytes and lymphatic endothelia, red), and lotus tetragonolobus lectin (LTL; proximal tubules, red) on serial frozen sections of intraomental kidney grafts from wild-type (WT) or $L t b r^{-/-}$mice over the course of 6 weeks. B: Bar graph showing PDPLN mean fluorescence intensity (MFI; plum bars) in glomeruli 2 weeks after transplantation in WT or Ltbr ${ }^{-/-}$ omentum, measured with ImageJ version 1.52a (each bar represents a glomerulus). Gray bars indicate the area of each glomerulus considered (ImageJ). C: Scatter dot plot with bar graphs \pm SD showing the length of $\mathrm{LTL}^{+}$tubules 2 weeks after transplantation in WT or $\mathrm{Ltbr}^{-/-}$omentum, measured across sections with ImageJ. Each dot represents a tubule. Power.t.test $=1$, sig.level $=0.05$. Data presented in this figure are representative of one of three technical replicates per sample. $n=10$ per group; 2 weeks: $n=1$ per group; 3 weeks: $n=4$ per group; 4 weeks: $n=1$ per group; 5 weeks: $n=1$ per group; 6 weeks: $n=3$ per group. ${ }^{* * * *} P<0.0001$. Scale bars $=100 \mu \mathrm{m}(\mathbf{A})$. 
kidneys engrafted in the wild-type omentum at the same stage. These cells were likely donor cells, which might have been trying to compensate for the missing host NIK function.

\section{LT $\beta R$ Plays a Role in Glomerular Adaptive Responses to $\mathrm{Nx}$}

Demonstration that LT $\beta R$ signaling is important for adult kidney repair would strengthen our hypothesis that kidney rebuilding approaches might benefit from the presence of a microenvironment that mediates LT $\beta R$ signals. To our knowledge, there are no studies that investigated LT $\beta R$ physiological role in kidney development or during compensatory growth.

The structure of $\mathrm{Ltbr}^{-1-}$ kidneys versus wild-type kidneys was first compared. LT $\beta$ R did not appear to be essential for kidney development during embryogenesis because $\mathrm{Ltbr}^{-1-}$ mice had normal kidneys with what appeared to be normal vascularization and structure (Supplemental Figure S7). This finding likely suggested differences between ectopic organogenesis and normal organogenesis. Indeed, although LT $\beta R$-angiogenetic signals appeared to be important for ectopic kidney organogenesis, in normal kidney organogenesis (which occurs mainly through vasculogenesis), LT $\beta R$ was not so crucial.

LT $\beta$ R relative contribution to compensatory renal growth after removal of one kidney was therefore investigated to corroborate the idea that not only was LT $\beta R$ signaling beneficial for glomerular vascularization/angiogenesis in lymphoid sites, but it also might play a role in glomerular adaptation to renal mass reduction.

Contralateral compensatory renal growth and increased renal function follows contralateral $\mathrm{Nx}$ in experimental models. ${ }^{33}$ Accordingly, although kidneys of wild-type and $L t b r^{-/-}$mice had similar weights before surgery, $L t b r^{-1-}$ mice showed significantly lower weights of their remnant kidneys 10 days after $\mathrm{Nx}$ (Figure 10A). The contralateral kidneys in wild-type mice showed glomerular enlargement as assessed in H\&E-stained sections (Figure 10B). These glomeruli often contained more erythrocytes, indicating an increase of renal blood flow. Differences in adaptive responses seemed to be confined to the glomerulus, because tubular epithelial cells from both mouse groups showed a similar widespread positivity for the Ki-67 proliferation marker (Figure 10C). These data indicated a more efficient glomerular cell response to unilateral Nx in the presence of functional LT $\beta R$ signaling.

\section{Discussion}

LNs and omenta can be used as bioreactors to grow organs. LN grafting is a unique approach pioneered by our laboratory, ${ }^{11,12,24,34,35}$ with no previous records in the literature. Conversely, the greater omentum has long been described as an ideal transplantation site mainly for its easy accessibility and large vascular network. ${ }^{36}$ Nevertheless, to our knowledge, cellular and molecular mechanisms that allow tissue and organ development within these locations have not been explored yet.
A

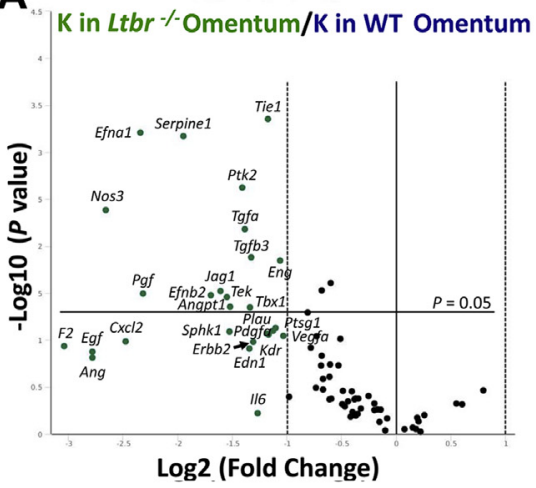

C

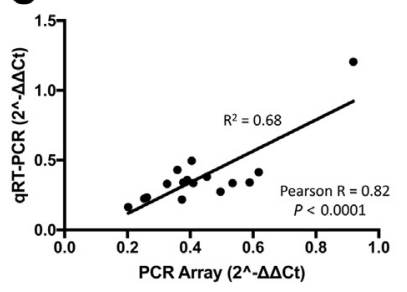

B

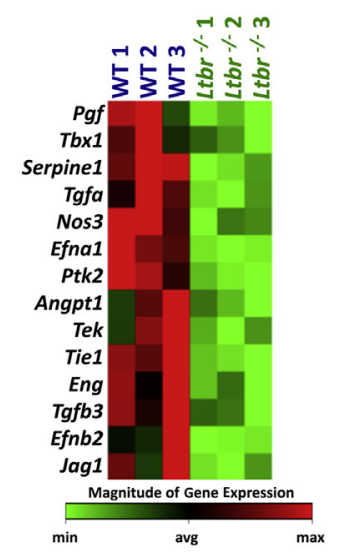

D

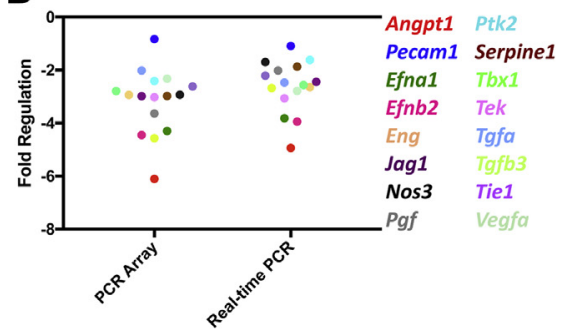

Figure 8 Down-regulation of pro-angiogenic genes in kidneys engrafted in the lymphotoxin- $\beta$ receptor $\left(\mathrm{Ltbr}^{-/-}\right)$omentum. A: The volcano plot displays genes differentially expressed in the kidneys engrafted in the $\mathrm{Ltbr}^{-/-}$omentum as compared with the kidneys engrafted in the wildtype (WT) omentum. Plotted along the $x$ axis is the mean of log2 fold-change; along the $y$ axis is the negative logarithm to the base 10 of the $P$ values. The vertical dashed lines and the horizontal line reflect the filtering criteria (fold change $\geq 2.0$ and $P$ values $\leq 0.05$ ). Green dots mark genes with decreased expression. B: Heatmap of the 14 statistically significant differentially expressed genes. C: Correlation between $\mathrm{RT}^{2}$ Profiler PCR Array and real-time PCR data. Relative expression levels determined by $\mathrm{RT}^{2}$ Profiler PCR Array ( $x$ axis; Qiagen) and real-time PCR ( $y$ axis) are plotted for a subset of 16 genes $\left(\mathrm{Ltbr}^{-/}{ }^{-}\right.$ 3 versus WT 3 ). Solid line indicates linear regression; both the squared and the Pearson correlation coefficients are given. D: Representative scatter plot $\left(\mathrm{Ltbr}^{-/-} 3\right.$ versus WT 3) showing the gene expression patterns (presented as fold change) of selected genes by $\mathrm{RT}^{2}$ Profiler PCR Array (PCR Array) and real-time PCR. 
A
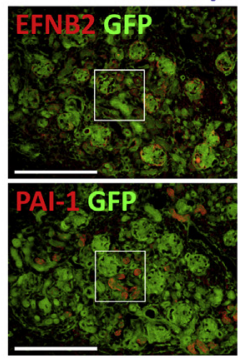

Kidney in Ltbr $\%$ Omentum
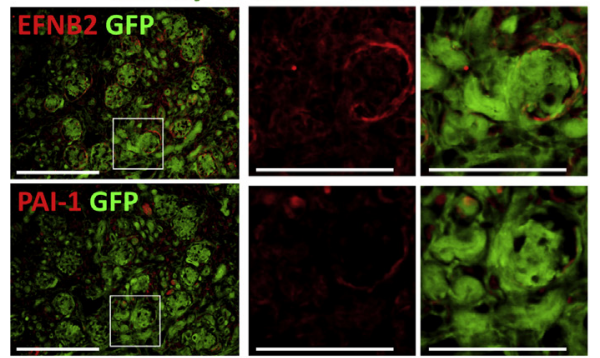

B

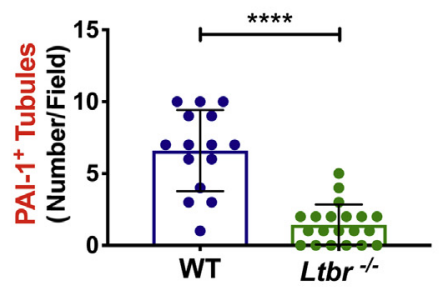

C
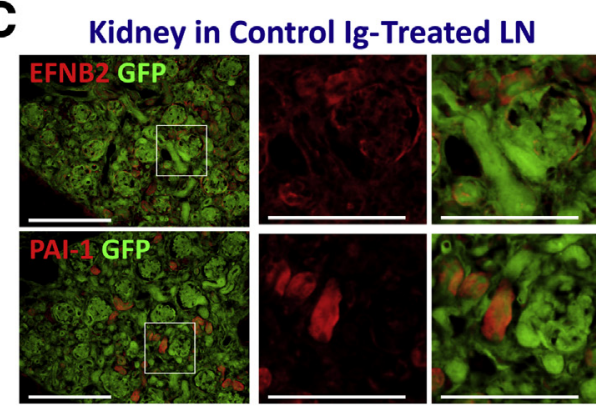

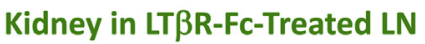
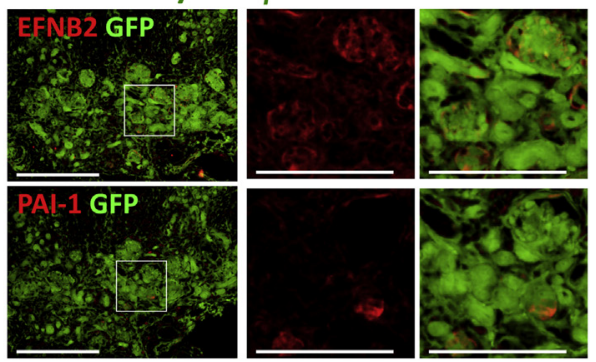

D
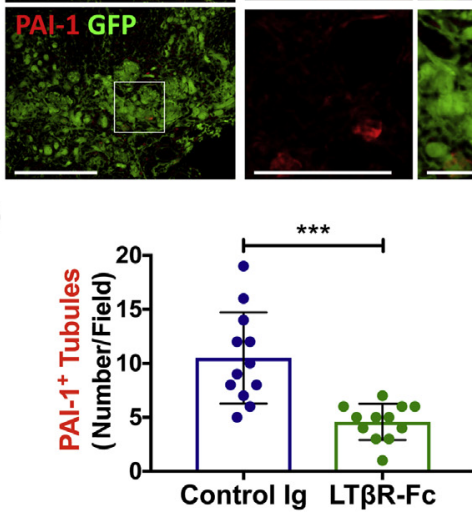

By transplanting kidney rudiments either in the LNs of mice undergoing LT $\beta R$ antagonist treatment or in the omenta of $\mathrm{Ltbr}^{-/-}$mice, it has been shown that host LT $\beta \mathrm{R}$ signals are crucial for obtaining a well-vascularized kidney graft. Indeed, defective host LT $\beta$ R signaling correlated with decreased expression of endothelial and angiogenic markers in the transplanted tissues as well as structural alterations. Although the number of glomerular endothelial cells expressing the LT $\beta R$ target NIK decreased in the absence of a functional LT $\beta R$, the results from the transplantation of kidney rudiments in the omenta of $\mathrm{Nik}^{-/-}$mice indicated that host NIK is not required for successful ectopic kidney organogenesis.

NIK and subsequent non-canonical NF- $\kappa \mathrm{B}$ signaling have been described to regulate both inflammation-induced and tumor-associated angiogenesis. ${ }^{17}$ From this perspective, kidney angiogenesis in lymphoid tissues shares features with pathologic angiogenesis. However, the progressive reduction in NIK-expressing cells that was observed in glomeruli engrafted in LNs and omenta seems to suggest a tight regulation of angiogenesis in the ectopic kidney grafts, whereby NIK likely elicits a transient signal. In the wild-type omentum, for example, NIK and the neoangiogenic marker CD105 shared a similar pattern, with decreased expressions in engrafted glomeruli starting as early as the second week after transplantation, a pattern different from that of the endothelial cell marker CD31, which was quite stable for 4 weeks. These data seem to suggest that NIK is required during the early stages of engraftment, coinciding with early angiogenesis and tissue vascular integration, but is no longer needed in later stages of kidney maturation. NIK-mediated signals, however, are not necessary for kidney organogenesis in lymphoid sites, because overall vascularization/angiogenesis was not affected in $\mathrm{Nik}^{-/-}$recipients.

Besides activating non-canonical NF- $\kappa \mathrm{B}$ pathway through NIK, ${ }^{16}$ LT $\beta$ R could also trigger the canonical $\mathrm{NF}-\kappa \mathrm{B}$ route to stimulate endothelial cells. ${ }^{37}$ Moreover, other angiogenic signals elicited by the donor tissue itself must not be underestimated, as indicated by the data of PAI-1 production by the kidney tubular cells. How host

\footnotetext{
Figure 9 Pro-angiogenic gene expression in kidneys engrafted in the omentum and lymph node (LN). A: Representative immunofluorescence stains for ephrin B2 (EFNB2; red) and plasminogen activator inhibitor-1 (PAI-1; red) on serial frozen sections of kidneys engrafted in wild-type (WT; top left panels) or lymphotoxin- $\beta$ receptor knockout ( $\mathrm{Ltbr}^{-/}$; bottom left panels) omenta ( 3 weeks after transplantation). Boxed areas are shown at higher magnification to the right. B: Scatter dot plot with bar graphs \pm SD presenting the number of PAI- $1^{+}$tubules per microscopic field of sections stained as in A. C: Representative immunofluorescence stains for EFNB2 and PAI-1 (red) on serial frozen sections of LN kidney grafts isolated from either mice treated with Control Ig (top left panels) or LT $\beta R$ Fc (bottom left panels) (3 weeks after transplantation). Boxed areas are shown at higher magnification to the right. D: Scatter dot plot with bar graphs \pm SD presenting the number of PAI- $1^{+}$tubules per microscopic field of sections stained as in $\mathbf{C}$. Data presented in this figure are representative of one of three technical replicates per sample. $n=3$ per group.

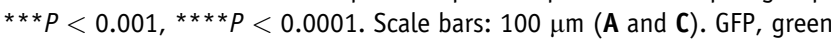
fluorescent protein.
} 
LT $\beta R$ signals can influence donor tissue production of PAI1 and possibly of other angiogenetic molecules remains unknown.

Kidney tissue integration after transplantation likely involves extensive vascular remodeling, whereby several players are involved, including endothelial cells, smooth muscle cells, and fibroblasts. To record these dynamic processes is technically demanding, but not impossible. Transplanting $\mathrm{GFP}^{+}$tissue in a wild-type recipient has been an effective method for visualizing the engrafted tissue later on, but the use of mice with constitutive or inducible endogenous fluorescent reporters, in combination with tracer injections and/or mosaic genetic recombination, ${ }^{38}$ would better allow characterizing the multicellular dynamics of angiogenesis and vascular remodeling in the transplanted tissues.

Three-dimensional reconstruction of the engrafted kidneys that was recently performed on human fetal kidneys engrafted in the $\mathrm{LN}^{24}$ would also allow us to better quantify the differences in tubular elongation across the experimental groups. Indeed, the current adopted strategy of $\mathrm{LTL}^{+}$tubule length quantification on two-dimensional sections cannot be considered accurate, because the apparent tubule length depends on the level at which the tissue was sectioned and observed. However, this strategy highlighted some important changes in the absence of a functional LT $\beta R$ signal that warrant future study.

Despite these limitations, the findings of host lymphoid stromal microenvironment contribution to ectopic kidney organogenesis seem to suggest implications for future development of new approaches to treat kidney disease. The renal sub-capsular space has served as the preferred site for kidney regeneration from embryonic metanephroi ${ }^{39,40}$ and recently kidney organoids. ${ }^{41-43}$ However, there is no convincing evidence that this approach can improve kidney function after a major injury. The inflammatory and fibrotic milieu of a host's diseased kidney could constitute an adverse environment for cell and tissue engraftment and ultimately function. Finally, clinical progress in renal subcapsular transplantation has lagged behind other techniques because of the inelastic and tight nature of the human renal sub-capsular space. ${ }^{44}$ Additional strategies to engineer functional kidneys include repopulating synthetic/decellularized scaffolds with kidney-specific cells and transplanting these constructs orthotopically. ${ }^{45}$ Unfortunately, these approaches have often failed to recapitulate native kidney functions because of inadequate blood supply. Indeed, despite efforts to endow the scaffolds with an endothelium, ${ }^{45}$ it is still not possible to generate vascularized and perfused renal tissues that produce urine and/or have
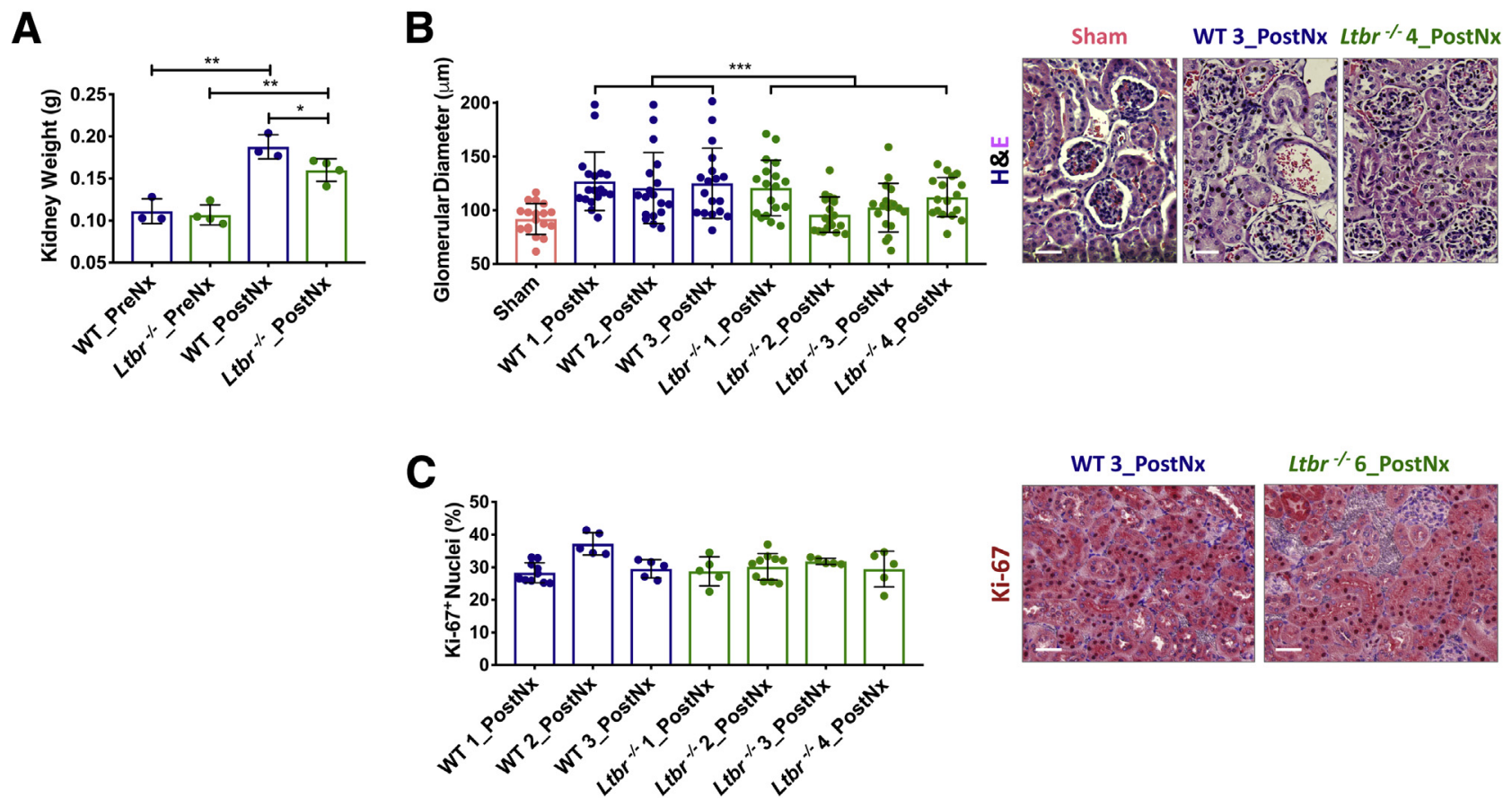

Figure 10 Lymphotoxin- $\beta$ receptor (LT $\beta R$ ) played a role in renal responses to nephrectomy ( $N x)$. A: Scatter dot plot with bar graph \pm SD showing kidney weights before and after Nx (PreNx and PostNx, respectively) in both wild-type (WT) and $L t b r^{-/-}$mice. Each dot represents a mouse. B: Left panel: Scatter dot plot with bar graph \pm SD showing glomerular diameters in WT and $L t b r^{-/-}$PostNx kidneys. Each dot represents a glomerulus. Right panel: Representative hematoxylin and eosin (H\&E) stain in Sham, WT_PostNx, or Ltbr ${ }^{-{ }_{-}}$_PostNx kidney paraffin sections. C: Left panel: Scatter dot plot with bar graph \pm SD showing the percentage of $\mathrm{Ki}-67^{+}$tubular cell nuclei from WT and $\mathrm{Ltbr}^{-/-}$PostNx kidneys. Each dot represents one stained section. Right panel: Representative immunohistochemistry for Ki-67 in WT and $\mathrm{Ltbr}^{-/}{ }^{-}$PostNx kidney paraffin sections (3-amino-9-ethylcarbazole, red). ${ }^{*} P<0.05,{ }^{* *} P<0.01$, ${ }^{* * *} P<0.001$. Scale bars: $50 \mu \mathrm{m}$ (B and $\left.\mathbf{C}\right)$. 
endocrine functions such as generation erythropoietin, ${ }^{46}$ necessary for erythropoiesis. ${ }^{47}$ Although reconstructing a fully functional kidney in the LN or the omentum for clinical purposes is challenging, stromal cells from these sites could be exploited to create an artificial scaffold for renal progenitor cells and thus provide an auxiliary aid during renal failure. A major concern about this approach might arise from the LT $\beta$ R documented role in kidney disease. Renal biopsies from lupus nephritis and IgA nephropathy patients showed elevated LT/LT $\beta R$ levels, and LT $\beta R$ inhibition improved renal function in a murine lupus model. ${ }^{48}$ LT $\beta$ R-Ig was efficacious when given from week 3 to 7 , whereas delaying the treatment until 5 weeks did not lead to significant results, highlighting a dynamic role for LT $\beta R$ across different stages of disease. ${ }^{48} \mathrm{LT} \beta \mathrm{R}$ signaling in lupus likely results from the formation of tertiary lymphoid organs. Unlike secondary lymphoid organs such as the LN, tertiary lymphoid organs arise in response to nonresolving inflammation over the course of several years. Therefore, although tertiary lymphoid organ-mediated LT $\beta R$ signaling leads to a prolonged, deregulated, and maladaptive response in injured kidneys that involves active inflammation and repeated unsuccessful attempts to repair the tissue, secondary lymphoid organ-mediated LT $\beta$ R signaling might be exploited to support kidney development. The finding that glomerular adaptive responses to loss of renal mass were impaired in the absence of a functional LT $\beta$ R indicated that LT $\beta R$ can participate to repair responses after injury, although we cannot rule out that these responses might become maladaptive over time.

In conclusion, although our results do not allow any unbiased conclusion on the precise cellular/molecular machineries that support kidney organogenesis in lymphoid sites, they point to an important function of the lymphoid stromal microenvironment in sustaining angiogenesis that warrants future investigation. Better understanding of the unique properties of this microenvironment might have implications for future engineering of functional organs.

\section{Acknowledgments}

We thank Hannah Liu and Adam DeDionisio for technical support with tissue sectioning and immunofluorescence/ immunohistochemistry staining and Lynda Guzik (McGowan Institute Flow Cytometry Facility) for help with flow cytometric profiling of lymph node and omental stromal cell populations and for proofreading the manuscript.

M.G.F. designed the study, performed most of the experiments, analyzed the data, prepared the figures, and wrote the manuscript; B.H. managed mouse colonies, performed the mouse surgeries, isolated omental stromal cells, and analyzed them by immunofluorescence; E.L. designed and supervised the study; all authors discussed the results and approved the final version of the manuscript.

\section{Supplemental Data}

Supplemental material for this article can be found at http://doi.org/10.1016/j.ajpath.2019.08.018.

\section{References}

1. Glassock RJ, Warnock DG, Delanaye P: The global burden of chronic kidney disease: estimates, variability and pitfalls. Nat Rev Nephrol 2017, 13:104-114

2. Neild GH: Life expectancy with chronic kidney disease: an educational review. Pediatr Nephrol 2017, 32:243-248

3. Glassock RJ, Pecoits-Filho R, Barberato SH: Left ventricular mass in chronic kidney disease and ESRD. Clin J Am Soc Nephrol 2009, 4 Suppl 1:S79-S91

4. Abecassis M, Bartlett ST, Collins AJ, Davis CL, Delmonico FL, Friedewald JJ, Hays R, Howard A, Jones E, Leichtman AB, Merion RM, Metzger RA, Pradel F, Schweitzer EJ, Velez RL, Gaston RS: Kidney transplantation as primary therapy for end-stage renal disease: a National Kidney Foundation/Kidney Disease Outcomes Quality Initiative (NKF/KDOQITM) conference. Clin J Am Soc Nephrol 2008, 3:471-480

5. Christians U, Klawitter J, Klawitter J, Brunner N, Schmitz V: Biomarkers of immunosuppressant organ toxicity after transplantation: status, concepts and misconceptions. Expert Opin Drug Metab Toxicol 2011, 7:175-200

6. Hammerman MR: Organogenesis of kidneys following transplantation of renal progenitor cells. Transpl Immunol 2004, 12: 229-239

7. Woolf AS, Palmer SJ, Snow ML, Fine LG: Creation of a functioning chimeric mammalian kidney. Kidney Int 1990, 38:991-997

8. Hyink DP, Tucker DC, St John PL, Leardkamolkarn V, Accavitti MA, Abrass CK, Abrahamson DR: Endogenous origin of glomerular endothelial and mesangial cells in grafts of embryonic kidneys. Am J Physiol 1996, 270:F886-F899

9. Rogers SA, Lowell JA, Hammerman NA, Hammerman MR: Transplantation of developing metanephroi into adult rats. Kidney Int 1998 $54: 27-37$

10. Matsumoto K, Yokoo T, Yokote S, Utsunomiya Y, Ohashi T, Hosoya T: Functional development of a transplanted embryonic kidney: effect of transplantation site. J Nephrol 2012, 25:50-55

11. Francipane MG, Lagasse E: The lymph node as a new site for kidney organogenesis. Stem Cells Transl Med 2015, 4:295-307

12. Francipane $\mathrm{MG}$, Lagasse $\mathrm{E}$ : Regenerating a kidney in a lymph node. Pediatr Nephrol 2016, 31:1553-1560

13. De Togni P, Goellner J, Ruddle NH, Streeter PR, Fick A Mariathasan S, Smith SC, Carlson R, Shornick LP, StraussSchoenberger J, Russell JH, Karr R, Chaplin DD: Abnormal development of peripheral lymphoid organs in mice deficient in lymphotoxin. Science 1994, 264:703-707

14. Futterer A, Mink K, Luz A, Kosco-Vilbois MH, Pfeffer K: The lymphotoxin beta receptor controls organogenesis and affinity maturation in peripheral lymphoid tissues. Immunity 1998, 9:59-70

15. Browning JL, Allaire N, Ngam-Ek A, Notidis E, Hunt J, Perrin S Fava RA: Lymphotoxin-beta receptor signaling is required for the homeostatic control of HEV differentiation and function. Immunity 2005, 23:539-550

16. Sun SC: Non-canonical NF-kappaB signaling pathway. Cell Res 2011, 21:71-85

17. Noort AR, van Zoest KP, Weijers EM, Koolwijk P, Maracle CX, Novack DV, Siemerink MJ, Schlingemann RO, Tak PP, Tas SW: NFkappaB-inducing kinase is a key regulator of inflammation-induced and tumour-associated angiogenesis. J Pathol 2014, 234:375-385 
18. Committee for the Update of the Guide for the Care and Use of Laboratory AnimalsNational Research Council: Guide for the Care and Use of Laboratory Animals: Eighth Edition. Washington, DC, National Academies Press, 2011

19. Boehm T, Scheu S, Pfeffer K, Bleul CC: Thymic medullary epithelial cell differentiation, thymocyte emigration, and the control of autoimmunity require lympho-epithelial cross talk via LTbetaR. J Exp Med 2003, 198:757-769

20. Franki AS, Van Beneden K, Dewint P, Hammond KJ, Lambrecht S, Leclercq G, Kronenberg M, Deforce D, Elewaut D: A unique lymphotoxin \{alpha\}beta-dependent pathway regulates thymic emigration of V $\{$ alpha\} 14 invariant natural killer T cells. Proc Natl Acad Sci U S A 2006, 103:9160-9165

21. Cardiff RD, Miller CH, Munn RJ: Manual hematoxylin and eosin staining of mouse tissue sections. Cold Spring Harb Protoc 2014, 2014:655-658

22. Haddad G, Zhabyeyev P, Farhan M, Zhu LF, Kassiri Z, Rayner DC, Vanhaesebroeck B, Oudit GY, Murray AG: Phosphoinositide 3kinase beta mediates microvascular endothelial repair of thrombotic microangiopathy. Blood 2014, 124:2142-2149

23. Fletcher AL, Malhotra D, Acton SE, Lukacs-Kornek V, BellemarePelletier A, Curry M, Armant M, Turley SJ: Reproducible isolation of lymph node stromal cells reveals site-dependent differences in fibroblastic reticular cells. Front Immunol 2011, 2:35

24. Francipane MG, Han B, Oxburgh L, Sims-Lucas S, Li Z, Lagasse E: Kidney-in-a-lymph node: a novel organogenesis assay to model human renal development and test nephron progenitor cell fates. J Tissue Eng Regen Med 2019, 13:1724-1731

25. Matsushima A, Kaisho T, Rennert PD, Nakano H, Kurosawa K, Uchida D, Takeda K, Akira S, Matsumoto M: Essential role of nuclear factor (NF)-kappaB-inducing kinase and inhibitor of kappaB (IkappaB) kinase alpha in NF-kappaB activation through lymphotoxin beta receptor, but not through tumor necrosis factor receptor I. J Exp Med 2001, 193:631-636

26. Chyou S, Ekland EH, Carpenter AC, Tzeng TC, Tian S, Michaud M, Madri JA, Lu TT: Fibroblast-type reticular stromal cells regulate the lymph node vasculature. J Immunol 2008, 181:3887-3896

27. Onder L, Danuser R, Scandella E, Firner S, Chai Q, Hehlgans T, Stein JV, Ludewig B: Endothelial cell-specific lymphotoxin-beta receptor signaling is critical for lymph node and high endothelial venule formation. J Exp Med 2013, 210:465-473

28. Upadhyay V, Fu YX: Lymphotoxin signalling in immune homeostasis and the control of microorganisms. Nat Rev Immunol 2013, 13: 270-279

29. Zhai Y, Guo R, Hsu TL, Yu GL, Ni J, Kwon BS, Jiang GW, Lu J, Tan J, Ugustus M, Carter K, Rojas L, Zhu F, Lincoln C, Endress G, Xing L, Wang S, Oh KO, Gentz R, Ruben S, Lippman ME, Hsieh SL, Yang D: LIGHT, a novel ligand for lymphotoxin beta receptor and TR2/HVEM induces apoptosis and suppresses in vivo tumor formation via gene transfer. J Clin Invest 1998, 102:1142-1151

30. Mauri DN, Ebner R, Montgomery RI, Kochel KD, Cheung TC, Yu GL, Ruben S, Murphy M, Eisenberg RJ, Cohen GH, Spear PG, Ware CF: LIGHT, a new member of the TNF superfamily, and lymphotoxin alpha are ligands for herpesvirus entry mediator. Immunity 1998, 8:21-30

31. Nangaku M: Mechanisms of tubulointerstitial injury in the kidney: final common pathways to end-stage renal failure. Intern Med 2004, 43:9-17

32. Tsujie M, Isaka Y, Ando Y, Akagi Y, Kaneda Y, Ueda N, Imai E, Hori M: Gene transfer targeting interstitial fibroblasts by the artificial viral envelope-type hemagglutinating virus of Japan liposome method. Kidney Int 2000, 57:1973-1980

33. Hayslett JP: Effect of age on compensatory renal growth. Kidney Int 1983, 23:599-602

34. Komori J, Boone L, DeWard A, Hoppo T, Lagasse E: The mouse lymph node as an ectopic transplantation site for multiple tissues. Nat Biotechnol 2012, 30:976-983

35. Francipane MG, Lagasse E: Maturation of embryonic tissues in a lymph node: a new approach for bioengineering complex organs. Organogenesis 2014, 10:323-331

36. Smink AM, Faas MM, de Vos P: Toward engineering a novel transplantation site for human pancreatic islets. Diabetes 2013, 62: $1357-1364$

37. Dejardin E, Droin NM, Delhase M, Haas E, Cao Y, Makris C, Li ZW, Karin M, Ware CF, Green DR: The lymphotoxin-beta receptor induces different patterns of gene expression via two NF-kappaB pathways. Immunity 2002, 17:525-535

38. Wang Y, Jin Y, Lavina B, Jakobsson L: Characterization of multicellular dynamics of angiogenesis and vascular remodelling by intravital imaging of the wounded mouse cornea. Sci Rep 2018, 8: 10672

39. Hammerman MR: Renal organogenesis from transplanted metanephric primordia. J Am Soc Nephrol 2004, 15:1126-1132

40. Imberti B, Corna D, Rizzo P, Xinaris C, Abbate M, Longaretti L, Cassis P, Benedetti V, Benigni A, Zoja C, Remuzzi G, Morigi M: Renal primordia activate kidney regenerative events in a rat model of progressive renal disease. PLoS One 2015, 10:e120235

41. Xinaris C, Benedetti V, Rizzo P, Abbate M, Corna D, Azzollini N, Conti S, Unbekandt M, Davies JA, Morigi M, Benigni A, Remuzzi G: In vivo maturation of functional renal organoids formed from embryonic cell suspensions. J Am Soc Nephrol 2012, 23:1857-1868

42. Sharmin S, Taguchi A, Kaku Y, Yoshimura Y, Ohmori T, Sakuma T, Mukoyama M, Yamamoto T, Kurihara H, Nishinakamura R: Human induced pluripotent stem cell-derived podocytes mature into vascularized glomeruli upon experimental transplantation. J Am Soc Nephrol 2016, 27:1778-1791

43. van den Berg CW, Ritsma L, Avramut MC, Wiersma LE, van den Berg BM, Leuning DG, Lievers E, Koning M, Vanslambrouck JM, Koster AJ, Howden SE, Takasato M, Little MH, Rabelink TJ: Renal subcapsular transplantation of PSC-derived kidney organoids induces neo-vasculogenesis and significant glomerular and tubular maturation in vivo. Stem Cell Reports 2018, 10:751-765

44. Sakata N, Yoshimatsu G, Kodama S: The spleen as an optimal site for islet transplantation and a source of mesenchymal stem cells. Int J Mol Sci 2018, 19:E1391

45. Song JJ, Guyette JP, Gilpin SE, Gonzalez G, Vacanti JP, Ott HC: Regeneration and experimental orthotopic transplantation of a bioengineered kidney. Nat Med 2013, 19:646-651

46. Obara N, Suzuki N, Kim K, Nagasawa T, Imagawa S, Yamamoto M: Repression via the GATA box is essential for tissue-specific erythropoietin gene expression. Blood 2008, 111:5223-5232

47. Adamson JW: Regulation of red blood cell production. Am J Med 1996, 101:4S-6S

48. Seleznik G, Seeger H, Bauer J, Fu K, Czerkowicz J, Papandile A, Poreci U, Rabah D, Ranger A, Cohen CD, Lindenmeyer M, Chen J, Edenhofer I, Anders HJ, Lech M, Wuthrich RP, Ruddle NH, Moeller MJ, Kozakowski N, Regele H, Browning JL, Heikenwalder M, Segerer S: The lymphotoxin beta receptor is a potential therapeutic target in renal inflammation. Kidney Int 2016, $89: 113-126$ 